\title{
Hepatoprotective Activity of Some Medicinal Plants in Sudan
}

\author{
Sumaia A. Ali $\mathbb{D}^{1}{ }^{1}$ Noha H. Sharief, ${ }^{2}$ and Yahya S. Mohamed ${ }^{3}$ \\ ${ }^{1}$ College of Veterinary Medicine, Sudan University of Science and Technology, Department of Veterinary Medicine and Surgery, \\ Khartoum, Sudan \\ ${ }^{2}$ Faculty of Veterinary Medicine, University of Khartoum, Khartoum, Sudan \\ ${ }^{3}$ Medicinal and Aromatic Plants Institute and Traditional Medicine, National Centre for Research, \\ Department of Phytochemistry and Taxonomy, Khartoum, Sudan
}

Correspondence should be addressed to Sumaia A. Ali; somiahadlool@yahoo.com

Received 30 April 2019; Revised 30 September 2019; Accepted 12 November 2019; Published 18 December 2019

Academic Editor: Ghee T. Tan

Copyright $\odot 2019$ Sumaia A. Ali et al. This is an open access article distributed under the Creative Commons Attribution License, which permits unrestricted use, distribution, and reproduction in any medium, provided the original work is properly cited.

Background. Liver disorders are common in Sudan and elsewhere. These are traditionally treated by medicinal plants especially in rural areas where they are widely available. Methods. This review was based on scientific research in hepatoprotective plants performed in Sudan for the period between 2001 and 2016 AD. Data collection was done through scientific evidence of local and international published data, theses, and publications from some libraries in Sudanese universities. Internet was also used to collect published data in different international scientific journals. Results. In this study, 21 plants from different families were reviewed for the hepatoprotective activity in Sudan. These plants are widely used in traditional medicine for their availability and cheap prices. All of these plants have been scientifically investigated through experimental animal models which confirmed their hepatoprotective activities. This was evaluated by measuring several parameters including liver markers (AST, ALT, ALP, total protein, albumin, and bilirubin) and histopathological investigation. Nineteen (90.5\%) of the herbal plants were found to possess significant hepatoprotective activity in animal models. Two (9.5\%) of the plants were devoid of this activity. The action of these plants is largely attributed to their phytoconstituents such as flavonoids, antioxidant, and anti-inflammatory effects. Conclusion. Sudanese herbs may offer novel alternatives to treat liver disorders. Yet determination of the active principle responsible for hepatoprotection needs to be investigated. Further studies on these plants are necessary to establish the efficacy, safety, and exact mechanism of action as a moral alternative in the treatment of liver disorders.

\section{Background}

Sudan possesses a unique variable climatic condition with abundant wealth of flora, cultivated or wild. These are used widely and effectively in folk medicine for treatment of various human and animal aliments, especially by natives in rural areas. In Sudan, medicinal folklore is passed from one generation to another but has not been properly documented $[1,2]$.

The liver is one of the most important body organs, owing to its multibiological functions in protein, lipid, and carbohydrate metabolism. Acute and chronic liver diseases constitute a global concern, and medical treatments for these diseases are often difficult to achieve and may have limited efficiency [3]. Liver diseases may be fatal posing a serious challenge to international public health [4]. Therefore, there has been considerable interest in complementary and alternative medicines for the treatment of hepatic disorders. Developing therapeutically effective agents from natural products may reduce the risk of toxicity when these drugs are used clinically [5].

The use of herbal medicines to treat liver diseases has increased worldwide, and this is due to the belief that herbal medicines are harmless and free from serious adverse reactions. In addition, they are available and easily obtained from nature. Moreover, the limited therapeutic choices and sometimes unsatisfactory therapeutic failure of modern medicine have increased the usage of alternative medicine including herbal preparations $[6,7]$.

Worldwide, a great number of remedial plants and their formulations have been claimed to have hepatoprotective activity. Around 160 phytoconstituents from 101 plants have 
been proved to possess liver-protecting activity [8]. Silymarin, a flavonolignan from "milk thistle" (Silybum marianum) plant is used almost exclusively for hepatoprotection [9]. It is known that $80 \%$ of the world population are using medicinal plants and $30 \%$ are prescribed by physicians [10]. In Sudan, it is estimated that over $90 \%$ of the population depend on plant-derived medicines [11].

Plants derived natural products such as flavonoids, terpenoids and sterols have received considerable attention in recent years due to their diverse pharmacological properties including antioxidant and hepatoprotective activity [12].

In vivo and in vitro evaluation models have been developed for the ability of the plants to prevent or cure liver toxicity in laboratory animals induced by various hepatotoxins [13]. The prevention of liver damage induced by $\mathrm{CCl}_{4}$ has widely been used as a marker of hepatoprotective activity of drugs in general [14].

Regardless of the fact that quite a number of Sudanese plant species are internationally renowned for high quality and commercial viability, they have not been fully exploited, probably due to lack of effective marketing system [2]. In the Folk medicine of Sudan, numerous plants were used effectively in the treatment of jaundice and different liver disorders, but in most cases their efficacy has not received any comprehensive scientific evaluation. In the present review, scientific literature evaluating the hepatoprotective activity of some Sudanese medicinal plants using different hepatotoxicity models has been discussed.

\section{Methods}

This review aimed at compiling data on hepatoprotective plants in Sudan published locally and internationally during the period between 2001 (starting point of scientific research about hepatoprotective herbs) and $2016 \mathrm{AD}$. In this review, the hepatoprotective herbs were listed according to scientific information obtained on their medicinal use for curing liver disorders, reported in publications and theses from some libraries in Sudanese universities. Internet was also used to collect published data in different international scientific journals through PubMed and Google Scholar search engines. Local traditional medicinal books such as $[1,2,15,16]$ were used to collect information about distribution and traditional uses of these plants. Literature search described the use of well-established experimental models (in-vivo and in-vitro) such as carbon tetrachloride $\left(\mathrm{CCl}_{4}\right)$-induced liver injury $[17,18]$ and paracetamol-induced liver injury [19] using experimental animals or cell line. Comparison is made between the efficacy of the herbal drug and that of conventional drugs used to treat liver disease particularly silymarin $[9,20]$. The liver enzymes aspartate transaminase (AST), alanine transaminase (ALT), alkaline phosphatase (APT), total bilirubin, total protein (TP), and albumin (Alb) were used as biomarkers to estimate the level of hepatocyte damage. Review also included the histopathological evaluation of the effect of the standard hepatotoxic agents compared with that of the plant extracts used as hepatoprotective agents.

Briefly, this review summarizes the scientific information of 21 hepatoprotective herbal drugs used in Sudan traditional medicine for the treatment of liver disorders including their botanical name, family, part of the plant used, local name, distribution, chemical constituents, traditional uses, the extracts used, the dosage of extracts, the model used, parameters estimated, histopathology, and the results of hepatoprotective studies on each plant. The possible mechanism of action of these hepatoprotective plants has been also discussed.

\section{Results}

Twenty-one Sudanese medicinal plants from different families were reviewed for the hepatoprotective activity in Sudan. These plants are widely used in traditional medicine especially in rural areas for their effectiveness, availability, and cheap prices.

These plants have been scientifically evaluated using different experimental models especially $\mathrm{CCl}_{4}$ and paracetamol to induce liver damage. These plants were assessed by measuring several parameters including liver markers (AST, ALT, ALP, total protein, albumin, and bilirubin) and histopathological investigation. Nineteen (90.5\%) of the medicinal plants were found to possess significant hepatoprotective activity in animal and cell line models. Two $(9.5 \%)$ of the plants were devoid of this activity.

Traditional plants commonly used to treat liver disorders in Sudan, their distribution, chemical composition, part of the plant used, method of preparation, and outcome of treatment are shown in Tables 1 and 2 and Figure 1.

3.1. Acacia mellifera (Vahl.), Fabaceae, Keka/Kitir. The hepatoprotective activity of $A$. mellifera leaves ethanolic extract and fractions were investigated against $\mathrm{DCFH}-$ (dichlorofluorescein) and $\mathrm{CCl}_{4}$-induced hepatotoxicity on cultured liver cells and rats [21]. DCFH-toxicated cells were recovered to about $100 \%$ with $100 \mu \mathrm{g} / \mathrm{ml}$ of $A$. mellifera (AM) crude extract, and supplementation with $200 \mu \mathrm{g} / \mathrm{ml}$ of this further enhanced the hepatocyte proliferation by about $20 \%$. Ethyl acetate, aqueous, and n-butanol fractions showed the most promising effective hepatoprotection, while hexane and dichloromethane fractions were devoid of this activity.

In $\mathrm{CCl}_{4}$ rat model, administration of $\mathrm{AM}$ ethanolic extract at a dose of 250 and $500 \mathrm{mg} / \mathrm{kg}$.b. wt orally and $\mathrm{CCl}_{4}$ in liquid paraffin $(1: 1,1.25 \mathrm{ml} / \mathrm{kg})$ intraperitoneally (IP) for three weeks significantly normalized alkaline phosphatase (ALP), bilirubin (Bil), cholesterol, triglyceride, and lipoprotein levels and elevated tissue nonprotein sulphhydryl and total protein. The histopathology showed that AM at a dose of $250 \mathrm{mg} / \mathrm{kg}$ revealed congested hepatic central veins with mild hepatocyte necrosis and fatty changes. Rats receiving $500 \mathrm{mg} / \mathrm{kg}$ of AM or silymarin showed normal hepatocytes and central veins.

The phytochemical screening of the fractions showed the presence of alkaloids, flavonoids, polyphenolic tannins, sterols, and saponins. The antioxidant activity of AM fractions was also performed against 1,1-diphenyl-2-picrylhydrazyl (DPPH) radical. The highest antioxidant activity was found in ethyl acetate and n-butanol followed by dichloromethane, hexane, and aqueous extracts. 
TABLE 1: Sudanese hepatoprotective herbs.

\begin{tabular}{|c|c|c|c|c|c|c|}
\hline Botanical names & Part used & Extracts used/dosage & Model(s) used & Parameters estimated & Histopathology & References \\
\hline $\begin{array}{l}\text { 1. Acacia mellifera } \\
\text { (Vahl) }\end{array}$ & Leaves & $\begin{array}{c}\text { Ethanol }(250 \text { and } \\
500 \mathrm{mg} / \mathrm{kg})\end{array}$ & $\mathrm{CCl}_{4}$-induced & $\begin{array}{l}\text { AST, ALT, GGT, ALP, } \\
\text { bilirubin, TP }\end{array}$ & Cured the tissue lesions & {$[21]$} \\
\hline $\begin{array}{l}\text { 2. Adansonia } \\
\text { digitata } \mathrm{L} \text {. }\end{array}$ & $\begin{array}{l}\text { Fruit's } \\
\text { pulp }\end{array}$ & $\begin{array}{c}\text { Methanol (100, } 200 \mathrm{mg} / \\
\mathrm{kg})\end{array}$ & $\mathrm{CCl}_{4}$-induced & $\begin{array}{l}\text { AST, ALT, ALP, } \\
\text { bilirubin }\end{array}$ & $\begin{array}{l}\text { Minimal necrosis and } \\
\text { regeneration of } \\
\text { hepatocytes compared } \\
\text { with } \mathrm{CCl}_{4}\end{array}$ & {$[22]$} \\
\hline $\begin{array}{l}\text { 3. Anogeissus } \\
\text { leiocarpus (DC) } \\
\text { Wall }\end{array}$ & Bark & Ethanol $(200 \mathrm{mg} / \mathrm{kg})$ & $\mathrm{CCl}_{4}$-induced & $\begin{array}{c}\text { AST, ALT, ALP, } \\
\text { bilirubin, total protein, } \\
\text { albumin }\end{array}$ & $\begin{array}{c}\text { Less hepatocyte } \\
\text { vacuolation and } \\
\text { cellular regeneration }\end{array}$ & {$[23]$} \\
\hline $\begin{array}{l}\text { 4. Argemone } \\
\text { Mexicana L. }\end{array}$ & $\begin{array}{l}\text { Aerial } \\
\text { parts }\end{array}$ & $\begin{array}{c}\text { Methanol, aqueous } \\
(100,200 \text {, and } 400 \mathrm{mg} / \\
\mathrm{kg})\end{array}$ & $\mathrm{CCl}_{4}$-induced & SGOT, SGPT, ALP & $\begin{array}{l}100 \mathrm{mg} / \mathrm{kg} \text { methanolic } \\
\text { extract more effective } \\
\text { in healing liver } \\
\text { parenchyma than other } \\
\text { doses; } 400 \mathrm{mg} / \mathrm{kg} \\
\text { aqueous extract showed } \\
\text { a good result than the } \\
\text { corresponding dose of } \\
\text { methanol extract }\end{array}$ & {$[24]$} \\
\hline $\begin{array}{l}\text { 5. Balanites } \\
\text { aegyptiaca L. }\end{array}$ & Bark & $\begin{array}{l}\text { Water, methanol (250 } \\
\text { and } 500 \mathrm{mg} / \mathrm{kg})\end{array}$ & $\mathrm{CCl}_{4}$-induced & $\begin{array}{l}\text { AST, ALT, ALP, } \\
\text { bilirubin }\end{array}$ & $\begin{array}{c}\text { Both extracts decrease } \\
\text { centrilobular } \\
\text { degenerative changes }\end{array}$ & {$[25]$} \\
\hline $\begin{array}{l}\text { 6. Cannabis } \\
\text { sativa } \mathrm{L} \text {. }\end{array}$ & Seeds & $\begin{array}{l}\text { Petroleum ether (1 and } \\
\qquad 0.5 \mathrm{ml} / \mathrm{kg})\end{array}$ & $\mathrm{CCl}_{4}$-induced & $\begin{array}{l}\text { AST, ALT, ALP, } \\
\text { bilirubin }\end{array}$ & $\begin{array}{l}1 \mathrm{ml} / \mathrm{kg} \text { : showed } \\
\text { scattered areas of } \\
\text { necrosis of the } \\
\text { hepatocytes } \\
0.5 \mathrm{ml} / \mathrm{kg} \text { : showed } \\
\text { slight areas of necrosis } \\
\text { and slight fatty change }\end{array}$ & {$[26]$} \\
\hline \multirow{3}{*}{$\begin{array}{l}\text { 7. Capparis decidua } \\
\text { (Forsk.) }\end{array}$} & Stems & $\begin{array}{l}\text { Aqueous, methanol } \\
(200 \text { and } 400 \mathrm{mg} / \mathrm{kg})\end{array}$ & $\mathrm{CCl}_{4}$-induced & $\begin{array}{l}\text { AST, ALT, ALP, } \\
\text { bilirubin }\end{array}$ & $\begin{array}{c}\text { Aqueous and } \\
\text { methanolic extracts } \\
\text { masked the liver fatty } \\
\text { changes }\end{array}$ & {$[7,27]$} \\
\hline & Branches & $\begin{array}{c}\text { Methanol }(12.5,25 \text {, and } \\
50 \mathrm{mg} / \mathrm{kg})\end{array}$ & $\begin{array}{l}\mathrm{CCl}_{4} \text {-induced, } \\
\text { paracetamol- } \\
\text { induced }\end{array}$ & $\begin{array}{l}\text { AST, ALT, ALP, } \\
\text { bilirubin, total protein, } \\
\text { albumin }\end{array}$ & 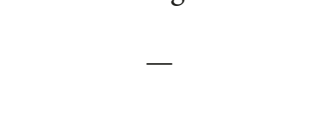 & {$[28]$} \\
\hline & Roots & Ethanol $(200 \mathrm{mg} / \mathrm{kg})$ & $\mathrm{CCl}_{4}$-induced & $\begin{array}{l}\text { AST, ALT, ALP, } \\
\text { bilirubin, total protein, } \\
\text { albumin }\end{array}$ & $\begin{array}{l}\text { Less hepatocyte } \\
\text { vacuolation and } \\
\text { cellular regeneration }\end{array}$ & {$[23]$} \\
\hline $\begin{array}{l}\text { 8. Combretum } \\
\text { hartmannianum } \\
\text { (Schweinf) }\end{array}$ & Leaves & $\begin{array}{c}\text { Methanol }(12.5,25 \text {, and } \\
50 \mathrm{mg} / \mathrm{kg})\end{array}$ & $\begin{array}{l}\mathrm{CCl}_{4} \text {-induced, } \\
\text { paracetamol- } \\
\text { induced. }\end{array}$ & $\begin{array}{c}\text { AST, ALT, ALP, } \\
\text { bilirubin, total protein, } \\
\text { albumin }\end{array}$ & - & {$[28]$} \\
\hline $\begin{array}{l}\text { 9. Dobera glabra } \\
\text { (Forsk.) }\end{array}$ & Leaves & $\begin{array}{l}\text { Aqueous methanol } \\
(200 \text { and } 400 \mathrm{mg} / \mathrm{kg})\end{array}$ & $\mathrm{CCl}_{4}$-induced & $\begin{array}{l}\text { AST, ALT, ALP, } \\
\text { bilirubin }\end{array}$ & $\begin{array}{c}\text { Diffuse centrilobuar } \\
\text { necrotic lesions in liver } \\
\text { cells }\end{array}$ & [29] \\
\hline \multirow{4}{*}{$\begin{array}{l}\text { 10. Khaya } \\
\text { senegalensis (Desr.) }\end{array}$} & Bark & $\begin{array}{c}\text { Aqueous (250 and } \\
500 \mathrm{mg} / \mathrm{kg})\end{array}$ & $\mathrm{CCl}_{4}$-induced & $\begin{array}{l}\text { AST, ALT, ALP, } \\
\text { bilirubin, total protein, } \\
\text { albumin }\end{array}$ & $\begin{array}{c}250 \mathrm{mg} / \mathrm{kg} \text { : showed } \\
\text { moderate necrosis of } \\
\text { the hepatocytes and } \\
\text { vacuolations } \\
500 \mathrm{mg} / \mathrm{kg} \text { : almost mild } \\
\text { fatty changes in livers }\end{array}$ & {$[30,31]$} \\
\hline & Bark & Ethanol (200 mg/kg) & $\mathrm{CCl}_{4}$-induced & $\begin{array}{c}\text { AST, ALT, ALP, } \\
\text { bilirubin, total protein, } \\
\text { albumin }\end{array}$ & $\begin{array}{l}\text { Mild histopathological } \\
\text { changes in the liver }\end{array}$ & {$[23]$} \\
\hline & Bark & $\begin{array}{c}\text { Methanol }(12.5,25 \text {, and } \\
50 \mathrm{mg} / \mathrm{kg}) .\end{array}$ & $\mathrm{CCl}_{4}$-induced & AST, ALT & - & {$[32]$} \\
\hline & Bark & Methanol $(50 \mathrm{mg} / \mathrm{kg})$ & $\begin{array}{l}\text { paracetamol- } \\
\text { induced }\end{array}$ & $\begin{array}{c}\text { AST, ALT, ALP, } \\
\text { bilirubin, total protein, } \\
\text { albumin }\end{array}$ & $\begin{array}{l}\text { Section of methanolic } \\
\text { extract-treated rats } \\
\text { showing few } \\
\text { vacuolation, reduced } \\
\text { sinusoidal dilatation, } \\
\text { and no congestion }\end{array}$ & [33] \\
\hline
\end{tabular}


TABLE 1: Continued.

\begin{tabular}{|c|c|c|c|c|c|c|}
\hline Botanical names & Part used & Extracts used/dosage & Model(s) used & Parameters estimated & Histopathology & References \\
\hline $\begin{array}{l}\text { 11. Kigelia africana } \\
\text { Lam. }\end{array}$ & Seeds & $\begin{array}{l}\text { Aqueous }(400 \mathrm{mg} / \mathrm{kg}) \\
\text { methanol }(100,200, \\
\text { and } 400 \mathrm{mg} / \mathrm{kg})\end{array}$ & $\mathrm{CCl}_{4}$-induced & AST, ALT, ALP & $\begin{array}{l}\text { Cytoplasmic fatty } \\
\text { vacuolation, } \\
\text { haemorrhages, and } \\
\text { necrosis of the } \\
\text { centrilobular } \\
\text { hepatocytes }\end{array}$ & [34] \\
\hline $\begin{array}{l}\text { 12. Lawsonia } \\
\text { inermis L. }\end{array}$ & Leaves & $\begin{array}{l}\text { Methanol (100 and } \\
200 \mathrm{mg} / \mathrm{kg})\end{array}$ & $\mathrm{CCl}_{4}$-induced & $\begin{array}{l}\text { AST, ALT, ALP, } \\
\text { bilirubin, total protein }\end{array}$ & $\begin{array}{l}\text { Showed minimal } \\
\text { necrosis and } \\
\text { regeneration of } \\
\text { hepatocytes compared } \\
\text { with } \mathrm{CCl}_{4}\end{array}$ & {$[35]$} \\
\hline $\begin{array}{l}\text { 13. Lepidium } \\
\text { sativum L. }\end{array}$ & Seeds & $\begin{array}{l}\text { Methanol (200 and } \\
\quad 400 \mathrm{mg} / \mathrm{kg})\end{array}$ & $\mathrm{CCl}_{4}$-induced & $\begin{array}{l}\text { AST, ALT, ALP, } \\
\text { bilirubin. }\end{array}$ & $\begin{array}{l}\text { Mild-to-moderate } \\
\text { changes in hepatocytes } \\
\text { in both doses }\end{array}$ & {$[36]$} \\
\hline $\begin{array}{l}\text { 14. Moringa } \\
\text { oleifera Lam. }\end{array}$ & Leaves & $\begin{array}{l}\text { Aqueous (200 and } \\
400 \mathrm{mg} / \mathrm{kg})\end{array}$ & $\mathrm{CCl}_{4}$-induced & $\begin{array}{l}\text { AST, ALT, ALP, } \\
\text { bilirubin }\end{array}$ & $\begin{array}{l}\text { Slight changes in liver } \\
\text { cells }\end{array}$ & {$[37]$} \\
\hline \multirow{3}{*}{ 15. Nigella sativa L. } & Seeds & $\begin{array}{l}\text { Methanol (250 and } \\
500 \mathrm{mg} / \mathrm{kg})\end{array}$ & $\mathrm{CCl}_{4}$-induced & AST, ALT, ALP & $\begin{array}{l}\text { Less centrilobular } \\
\text { vacuolation and } \\
\text { inflammatory cell } \\
\text { infiltration. }\end{array}$ & {$[38]$} \\
\hline & Seeds & $\begin{array}{c}\text { Aqueous (250 and } \\
500 \mathrm{mg} / \mathrm{kg})\end{array}$ & $\mathrm{CCl}_{4}$-induced & $\begin{array}{c}\text { AST, ALT, ALP, } \\
\text { bilirubin, total protein }\end{array}$ & $\begin{array}{c}\text { Less hepatocyte } \\
\text { changes }\end{array}$ & {$[30]$} \\
\hline & Seeds & $\begin{array}{l}\text { Methanol }(25,50,75 \text {, } \\
\text { and } 100 \mathrm{mg} / \mathrm{mL}) \text { in } \\
\text { vitro } \\
\text { Methanol }(100,300 \text {, } \\
\text { and } 900 \mathrm{mg} / \mathrm{kg}) \text { in vivo }\end{array}$ & $\begin{array}{l}\text { Paracetamol- } \\
\text { induced }\end{array}$ & $\begin{array}{l}\text { Blood ions, ALT, AST, } \\
\text { ALP, antioxidant } \\
\text { enzymes, e.g., SOD, } \\
\text { GSH, and MDA }\end{array}$ & $\begin{array}{c}\text { Improvement of lesions } \\
\text { observed in } \mathrm{CCl}_{4} \text { such } \\
\text { as necrosis, loss of } \\
\text { hepatocytes } \\
\text { architecture, and } \\
\text { cellular infiltration }\end{array}$ & {$[39]$} \\
\hline $\begin{array}{l}\text { 16. Occimum } \\
\text { basilicum L. }\end{array}$ & $\begin{array}{l}\text { Whole } \\
\text { plant }\end{array}$ & Ethanol (200 mg/kg) & $\mathrm{CCl}_{4}$-induced & $\begin{array}{l}\text { AST, ALT, ALP, } \\
\text { bilirubin, total protein, } \\
\text { albumin }\end{array}$ & $\begin{array}{l}\text { Less vacuolated } \\
\text { hepatocytes and } \\
\text { cellular regeneration }\end{array}$ & {$[40]$} \\
\hline $\begin{array}{l}\text { 17. Phoenix } \\
\text { dactylifera Linn. }\end{array}$ & $\begin{array}{l}\text { Pollen } \\
\text { grain } \\
\text { powder }\end{array}$ & $\begin{array}{l}\text { Water }(250 \text { and } \\
500 \mathrm{mg} / \mathrm{kg})\end{array}$ & $\mathrm{CCl}_{4}$-induced & AST, ALT, ALP & $\begin{array}{l}\text { Mild changes in } \\
\text { hepatocytes }\end{array}$ & {$[41]$} \\
\hline $\begin{array}{l}\text { 18. Raphanus } \\
\text { sativus L. }\end{array}$ & Seeds & $\begin{array}{l}\text { Methanol, water (200 } \\
\text { and } 400 \mathrm{mg} / \mathrm{kg})\end{array}$ & $\mathrm{CCl}_{4}$-induced & $\begin{array}{l}\text { AST, ALT, ALP, } \\
\text { bilirubin }\end{array}$ & $\begin{array}{c}\text { Mild-to-moderate } \\
\text { changes in hepatocytes } \\
\text { in both doses }\end{array}$ & {$[42]$} \\
\hline $\begin{array}{l}\text { 19. Solanum } \\
\text { nigrum L. }\end{array}$ & $\begin{array}{l}\text { Whole } \\
\text { plant }\end{array}$ & $\begin{array}{l}\text { Water, methanol (250 } \\
\text { and } 500 \mathrm{mg} / \mathrm{kg})\end{array}$ & $\mathrm{CCl}_{4}$-induced & $\begin{array}{l}\text { AST, ALT, ALP, } \\
\text { bilirubin }\end{array}$ & $\begin{array}{l}\text { Water: small vacuoles } \\
\text { were seen in } \\
\text { centrilobular } \\
\text { hepatocytes. In } \\
\text { methanol, slight } \\
\text { changes in the fat } \\
\text { content of hepatocytes } \\
\text { were seen. }\end{array}$ & {$[43]$} \\
\hline $\begin{array}{l}\text { 20. Sterculia } \\
\text { Setigera Del. }\end{array}$ & Bark & $\begin{array}{l}\text { Ethanol, ethyl acetate } \\
(200 \text { and } 400 \mathrm{mg} / \mathrm{kg})\end{array}$ & $\mathrm{CCl}_{4}$-induced & $\begin{array}{l}\text { AST, ALT, ALP, } \\
\text { bilirubin, total protein, } \\
\text { albumin }\end{array}$ & $\begin{array}{l}\text { Showed no stenosis } \\
\text { (fatty changes in } \\
\text { hepatocytes) and } \\
\text { perivenular fibrosis } \\
\text { compared with the } \\
\mathrm{CCl}_{4} \text { group. }\end{array}$ & {$[44]$} \\
\hline $\begin{array}{l}\text { 21. Tamarindus } \\
\text { indica } \mathrm{L} \text {. }\end{array}$ & $\begin{array}{l}\text { Fruit's } \\
\text { pulp }\end{array}$ & Ethanol (150 mg/kg) & $\mathrm{CCl}_{4}$-induced & $\begin{array}{l}\text { AST, ALT, ALP, } \\
\text { bilirubin }\end{array}$ & $\begin{array}{c}\text { Showed slight fatty } \\
\text { changes }\end{array}$ & {$[45]$} \\
\hline
\end{tabular}

The hepatoprotective activity of the plant may be attributed to the phenolic compounds, flavonoids, and saponins [61-64]. Hepatoprotective activity of flavonoids is due to their ability to scavenge free radicals [62].
3.2. Adansonia digitata L., Malvaceae, Baobab Tree. The hepatoprotective effect of the methanolic extract of Adansonia digitata L. fruit pulp (100 and $200 \mathrm{mg} / \mathrm{kg}$ ) was investigated against $\mathrm{CCl}_{4}$-induced hepatotoxicity in rats. Silymarin $(25 \mathrm{mg} /$ 
TABLE 2: Families, local names, distribution, traditional uses, and chemical constituents of hepatoprotective plants.

\begin{tabular}{|c|c|c|c|c|c|c|}
\hline Plants & Family & Local name & $\begin{array}{l}\text { Distribution in } \\
\text { Sudan }\end{array}$ & Traditional uses & Chemical constituents & References \\
\hline $\begin{array}{l}\text { 1. Acacia } \\
\text { mellifera (Vahl) }\end{array}$ & Fabaceae & Keka/kitir & $\begin{array}{l}\text { Northern and } \\
\text { Central Sudan }\end{array}$ & $\begin{array}{l}\text { Bowel problems, } \\
\text { stomach trouble, } \\
\text { cold, treatment for } \\
\text { malaria and } \\
\text { inflammation }\end{array}$ & $\begin{array}{l}\text { Alkaloids, flavonoids, } \\
\text { tannins, sterols, and } \\
\text { saponins }\end{array}$ & {$[2,21,46]$} \\
\hline $\begin{array}{l}\text { 2. Adansonia } \\
\text { digitata } \mathrm{L} \text {. }\end{array}$ & Malvaceae & $\begin{array}{l}\text { Baobab tree, } \\
\text { tabaldi, fruit } \\
\text { (gonglize) }\end{array}$ & $\begin{array}{l}\text { Widespread and } \\
\text { throughout } \\
\text { Northern and } \\
\text { Central Sudan, } \\
\text { Kordofan, Darfur }\end{array}$ & $\begin{array}{l}\text { Immunostimulant, } \\
\text { anti-inflammatory, } \\
\text { analgesic, pesticide, } \\
\text { antipyretic, } \\
\text { febrifuge, and } \\
\text { astringent in the } \\
\text { treatment of } \\
\text { diarrhea }\end{array}$ & $\begin{array}{l}\text { Terpenoids, flavonoids, } \\
\text { steroids, vitamins, } \\
\text { amino acids, } \\
\text { carbohydrates, and } \\
\text { lipids }\end{array}$ & {$[15,16,22,47]$} \\
\hline $\begin{array}{l}\text { 3. Argemone } \\
\text { Mexicana L. }\end{array}$ & Papaveraceae & Khash khash & Northern Sudan & $\begin{array}{c}\text { Analgesic, } \\
\text { antispasmodic, } \\
\text { possibly } \\
\text { hallucinogenic, and } \\
\text { sedative, tumors, } \\
\text { warts, skin diseases, } \\
\text { inflammations, } \\
\text { rheumatism, } \\
\text { jaundice, leprosy, } \\
\text { piles, warm } \\
\text { infestations, and } \\
\text { dysentery }\end{array}$ & $\begin{array}{l}\text { Alkaloids as berberine, } \\
\text { protopine, } \\
\text { sarguinarine, optisine, } \\
\text { and chelerytherine; the } \\
\text { seed oil contains } \\
\text { myristic, palmitic, } \\
\text { oleic, and linoleic acids; } \\
\text { flavonoids, glycosides, } \\
\text { sterols, and phenolic } \\
\text { compounds (tannins) }\end{array}$ & {$[24,48,49]$} \\
\hline $\begin{array}{l}\text { 4. Anogeissus } \\
\text { leiocarpus (DC. } \\
\text { Wall) }\end{array}$ & Combretaceae & Sahib & $\begin{array}{c}\text { Kassala, } \\
\text { Kordofan, Darfur }\end{array}$ & $\begin{array}{l}\text { Parasitic diseases } \\
\text { and dysentery }\end{array}$ & $\begin{array}{c}\text { Rich in derivatives of } \\
\text { ellagic acid, } \\
\text { polyalcohol sorbitol, } \\
\text { terpenoids ( } \alpha \text {-amyrin, } \\
\beta \text {-amyrin and } \\
\beta \text {-sitosterol), and traces } \\
\text { of alkaloids }\end{array}$ & {$[15,23,50,51]$} \\
\hline $\begin{array}{l}\text { 5. Balanites } \\
\text { aegyptiaca } \mathrm{L} .\end{array}$ & Balanitaceae & $\begin{array}{l}\text { Hegleig tree } \\
(\text { Ar), laloub } \\
\text { fruit }\end{array}$ & $\begin{array}{l}\text { Widespread. and } \\
\text { throughout } \\
\text { Northern and } \\
\text { Central Sudan }\end{array}$ & $\begin{array}{l}\text { Jaundice, liver } \\
\text { disorders, and } \\
\text { spleen problems; } \\
\text { the leaves and } \\
\text { branches are used } \\
\text { as a fumigant for } \\
\text { rheumatism; fruits } \\
\text { are used aginst } \\
\text { constipation and as } \\
\text { an antidiabetic }\end{array}$ & $\begin{array}{l}\text { The root contains } \\
\text { rotenone and } \\
\text { yamogenin; bark } \\
\text { contains steroidal } \\
\text { sapogenins }\end{array}$ & {$[15,16,25,52,53]$} \\
\hline $\begin{array}{l}\text { 6. Cannabis } \\
\text { sativa } \mathrm{L} \text {. }\end{array}$ & Cannabaceae & $\begin{array}{l}\text { Bango, } \\
\text { hashish. }\end{array}$ & $\begin{array}{c}\text { Cultivated in } \\
\text { various areas in } \\
\text { Sudan }\end{array}$ & $\begin{array}{c}\text { Spasmolytic, } \\
\text { hypnotic, analgesic } \\
\text { to treat rabies, } \\
\text { cholera, } \\
\text { rheumatism, } \\
\text { epilepsy, and } \\
\text { tetanus; anti- } \\
\text { inflammatory }\end{array}$ & $\begin{array}{c}\text { Cannabinoids } \\
\text { (tetrahydrocannabinol } \\
(\mathrm{THC}) \text { ), cannabidiol } \\
(\mathrm{CBD}) \text { and cannabinol } \\
(\mathrm{CBN}) \text { are the most } \\
\text { prevalent natural } \\
\text { cannabinoids) }\end{array}$ & {$[26]$} \\
\hline $\begin{array}{l}\text { 7. Capparis } \\
\text { decidua (Forsk.) }\end{array}$ & Capparaceae & Tundub & $\begin{array}{l}\text { Northern and } \\
\text { central Sudan; } \\
\text { Western and } \\
\text { eastern Sudan }\end{array}$ & $\begin{array}{c}\text { Jaundice, } \\
\text { antirheumatic } \\
\text { diuretics, antigout, } \\
\text { anti-inflammatory, } \\
\text { astringent, } \\
\text { stomachic, laxative, } \\
\text { antidote, and used } \\
\text { for skin diseases }\end{array}$ & $\begin{array}{l}\text { Alkaloids, saponins, } \\
\text { flavonoids, tannins, } \\
\text { sterols, cyanogenic } \\
\text { glycosides and } \\
\text { cumarins }\end{array}$ & {$[7,15,16,27]$} \\
\hline
\end{tabular}


TABle 2: Continued.

\begin{tabular}{|c|c|c|c|c|c|c|}
\hline Plants & Family & Local name & $\begin{array}{l}\text { Distribution in } \\
\text { Sudan } \\
\end{array}$ & Traditional uses & Chemical constituents & References \\
\hline $\begin{array}{l}\text { 8. Combretum } \\
\text { hartmannianum } \\
\text { (Schweinf) }\end{array}$ & Combretaceae & $\begin{array}{l}\text { Habiel, } \\
\text { Habeel Al } \\
\text { Gabal }\end{array}$ & $\begin{array}{l}\text { El-Jebelein. } \\
\text { White Nile, } \\
\text { Kordofan, } \\
\text { Darfur, Blue Nile }\end{array}$ & $\begin{array}{l}\text { Boiled leaves used } \\
\text { to cure ascites; bark } \\
\text { and leaves extract } \\
\text { are used for } \\
\text { jaundice }\end{array}$ & $\begin{array}{l}\text { Alkaloids, flavonoids, } \\
\text { tannins hydrocyanic } \\
\text { acid, and phenanthrene } \\
\text { derivatives }\end{array}$ & {$[1,2,15,28]$} \\
\hline $\begin{array}{l}\text { 9. Dobera glabra } \\
\text { (Forsk.) }\end{array}$ & Salvadoraceae & Al meikah & $\begin{array}{l}\text { Widespread. } \\
\text { Northern Sudan, } \\
\text { Kordofan, } \\
\text { Darfur, } \\
\text { Khartoum } \\
\end{array}$ & $\begin{array}{l}\text { Ophthalmic } \\
\text { problems }\end{array}$ & $\begin{array}{l}\text { Alkaloids, saponins, } \\
\text { flavonoids, tannins, } \\
\text { sterols, cyanogenic } \\
\text { glycosides and } \\
\text { cumarins } \\
\end{array}$ & {$[2,15,29]$} \\
\hline $\begin{array}{l}\text { 10. Khaya } \\
\text { senegalensis } \\
\text { (Desr.) }\end{array}$ & Meliaceae & $\begin{array}{l}\text { Mahogany } \\
\text { tree }\end{array}$ & Darfur & $\begin{array}{c}\text { Dysentery, } \\
\text { diarrhea, and } \\
\text { wound infections, } \\
\text { fever, and remedy } \\
\text { to treat syphilis; the } \\
\text { bark is used in } \\
\text { jaundice, scorpion } \\
\text { bites, allergies }\end{array}$ & $\begin{array}{l}\text { Saponins, tannins, } \\
\text { alkaloids, glycosides, } \\
\text { steroids, terpenoids } \\
\text { flavonoids, and } \\
\text { phenolic compounds }\end{array}$ & {$[23,54]$} \\
\hline $\begin{array}{l}\text { 11. Kigelia } \\
\text { africana Lam. }\end{array}$ & Bignoniaceous & $\begin{array}{l}\text { Umm } \\
\text { Shutour }\end{array}$ & $\begin{array}{l}\text { Central Sudan, } \\
\text { Kassala, } \\
\text { Kordofan, Blue } \\
\text { Nile }\end{array}$ & $\begin{array}{c}\text { Bark is used for } \\
\text { ulcers treatment or } \\
\text { for treatment of } \\
\text { pneumonia and } \\
\text { malaria }\end{array}$ & $\begin{array}{l}\text { Verminosides and } \\
\text { iridoids and series of } \\
\text { polyphenols such as } \\
\text { verbascoside }\end{array}$ & {$[2,15,34]$} \\
\hline $\begin{array}{l}\text { 12. Lawsonia } \\
\text { inermis L. }\end{array}$ & Lythraceae & Henna & $\begin{array}{l}\text { Widespread } \\
\text { especially in } \\
\text { Northern and } \\
\text { Central Sudan }\end{array}$ & $\begin{array}{c}\text { Astringent, } \\
\text { hypotensive, } \\
\text { sedative, and } \\
\text { against headache, } \\
\text { jaundice, leprosy, } \\
\text { and skin diseases }\end{array}$ & $\begin{array}{l}\text { Naphthoquinone, } \\
\text { phenolic derivatives, } \\
\text { coumarins, xanthones, } \\
\text { tannins, flavonoids, } \\
\text { aliphatic components, } \\
\text { triterpenes, sterols } \\
\text { glucose, gallic acid, } \\
\text { amino acids, mannitol, } \\
\text { trace elements, and } \\
\text { minerals }\end{array}$ & {$[2,15,35]$} \\
\hline $\begin{array}{l}\text { 13. Lepidium } \\
\text { sativum L. }\end{array}$ & Brassicaceae & $\begin{array}{l}\text { Pepper cress } \\
\text { or ELRshad }\end{array}$ & Central Sudan & $\begin{array}{c}\text { Gastrointestinal } \\
\text { disorders, arthritis, } \\
\text { and inflammatory } \\
\text { disorders dysentery } \\
\text { and diarrhea }\end{array}$ & $\begin{array}{c}\text { Triterpenes, alkaloids, } \\
\text { flavanoids, tannins, } \\
\text { coumarins, and } \\
\text { saponins }\end{array}$ & {$[2,36]$} \\
\hline $\begin{array}{l}\text { 14. Moringa } \\
\text { oleifera Lam. }\end{array}$ & Moringiaceae & Al-rawag & $\begin{array}{l}\text { Widespread, } \\
\text { Northern and } \\
\text { Central Sudan }\end{array}$ & $\begin{array}{l}\text { Liver disease, lipid } \\
\text { disorders, arthritis, } \\
\text { and inflammatory } \\
\text { disorders; seeds } \\
\text { used to clean water } \\
\text { for drinking }\end{array}$ & $\begin{array}{l}\text { Alkaloids, saponins, } \\
\text { flavonoids, tannins, } \\
\text { sterols, glycosides, and } \\
\text { cumarins }\end{array}$ & {$[2,15,37]$} \\
\hline $\begin{array}{l}\text { 15. Nigella } \\
\text { sativa L. }\end{array}$ & Ranunculaceae & $\begin{array}{l}\text { Black seed, } \\
\text { black cumin, } \\
\text { or habat } \\
\text { elbarka }\end{array}$ & $\begin{array}{l}\text { Northern Sudan, } \\
\text { Darfur (Melit and } \\
\text { Jebel Marra) }\end{array}$ & $\begin{array}{l}\text { Liver tonics, } \\
\text { digestive, anti- } \\
\text { inflammatory, } \\
\text { immunostimulant, } \\
\text { and remedy for } \\
\text { jaundice, } \\
\text { antidiabetics }\end{array}$ & $\begin{array}{l}\text { Thymoquinone, } \\
\text { thymohydro quinine, } \\
\text { dithymoquinone, p- } \\
\text { cymene, carvacrol, and } \\
\text { 4-terpineol }\end{array}$ & {$[38]$} \\
\hline
\end{tabular}


TABLE 2: Continued.

\begin{tabular}{|c|c|c|c|c|c|c|}
\hline Plants & Family & Local name & $\begin{array}{l}\text { Distribution in } \\
\text { Sudan }\end{array}$ & Traditional uses & Chemical constituents & References \\
\hline $\begin{array}{l}\text { 16. Occimum } \\
\text { basilicum L. }\end{array}$ & Lamiaceae & Alryhan & $\begin{array}{l}\text { Widespread } \\
\text { throughout } \\
\text { Northern and } \\
\text { Central Sudan }\end{array}$ & $\begin{array}{c}\text { Jaundice, stomach } \\
\text { complaints, fever, } \\
\text { cough, and gout, } \\
\text { diuretic, } \\
\text { aphrodisiac, and } \\
\text { antidysenteric } \\
\text { actions; the seeds } \\
\text { are used as } \\
\text { demulcents }\end{array}$ & $\begin{array}{l}\text { Glycoside, gums, } \\
\text { mucilage, proteins, } \\
\text { amino acids, tannins, } \\
\text { phenolic compound, } \\
\text { triterpenoids steroids, } \\
\text { sterols, saponins, } \\
\text { flavones, and } \\
\text { flavonoids } \\
\text { Linalol methylchavikol, } \\
\text { methylcinnamat, and } \\
\text { linolen, essential oil, } \\
\text { rosmarinic acid, citral, } \\
\text { eugenol, and geraniol }\end{array}$ & {$[16,40,55]$} \\
\hline $\begin{array}{l}\text { 17. Phoenix } \\
\text { dactylifera Linn. }\end{array}$ & Palmae & $\begin{array}{l}\text { Date, date } \\
\text { palm }\end{array}$ & $\begin{array}{l}\text { Northern Sudan, } \\
\text { most parts of the } \\
\text { Sudan }\end{array}$ & $\begin{array}{l}\text { Sexual incapacity } \\
\text { and weakness }\end{array}$ & $\begin{array}{c}\text { Carbohydrates, } \\
\text { alkaloids, steroids, } \\
\text { flavonoids, vitamins } \\
\text { tannins, and phenolic } \\
\text { acids }\end{array}$ & {$[15,41,56]$} \\
\hline $\begin{array}{l}\text { 18. Raphanus } \\
\text { sativus L. }\end{array}$ & Cruciferae & Alfgel. & Widespread & $\begin{array}{l}\text { Hepatoprotective, } \\
\text { bacterial and viral } \\
\text { infections, } \\
\text { inflammation }\end{array}$ & $\begin{array}{c}\text { Flavonoids, saponins, } \\
\text { cumarins, and } \\
\text { alkaloids }\end{array}$ & {$[42]$} \\
\hline $\begin{array}{l}\text { 19. Solanum } \\
\text { nigrum L. }\end{array}$ & Solanaceae & Enab eldib & $\begin{array}{l}\text { Poisonous plants } \\
\text { in the Sudan }\end{array}$ & $\begin{array}{l}\text { Inflammatory } \\
\text { disorders, } \\
\text { rheumatism and } \\
\text { swellen joints, } \\
\text { hepatomegaly, } \\
\text { splenomegaly, } \\
\text { edema, gonorrhea }\end{array}$ & $\begin{array}{l}\text { Gallic acid, catechin, } \\
\text { protocatechuic acid, } \\
\text { caffeic acid, } \\
\text { epicatechin, rutin, and } \\
\text { naringenin }\end{array}$ & {$[43,57]$} \\
\hline $\begin{array}{l}\text { 20. Sterculia } \\
\text { setigera Del. }\end{array}$ & Sterculiaceae & Terter & $\begin{array}{c}\text { Blue Nile, } \\
\text { Kassala, } \\
\text { Kordofan, Nuba } \\
\text { mountains, } \\
\text { Darfur and red } \\
\text { Sea hill regions }\end{array}$ & $\begin{array}{l}\text { Jaundice and } \\
\text { bilharzia }\end{array}$ & $\begin{array}{l}\text { Saponins, steroidal, } \\
\text { sterols, and flavonoids } \\
\text { Tannins, saponins, } \\
\text { cardiac glycosides, and } \\
\text { anthraquinone }\end{array}$ & {$[15,44,58]$} \\
\hline $\begin{array}{l}\text { 21. Tamarindus } \\
\text { indica } \mathrm{L} \text {. }\end{array}$ & Caesalpinaceae & Aradaib. & Central Sudan & $\begin{array}{l}\text { To treat fever, } \\
\text { postpartum } \\
\text { remedy, and } \\
\text { measles }\end{array}$ & $\begin{array}{c}\text { Tannins and alkaloids } \\
\text { and low concentration } \\
\text { of flavonoids and } \\
\text { saponins }\end{array}$ & {$[15,45,59,60]$} \\
\hline
\end{tabular}

$\mathrm{kg}$ ) was used as a reference drug. The two doses of $A$. digitata showed dose-dependant hepatoprotective effects on $\mathrm{CCl}_{4^{-}}$ induced hepatotoxicity in rats. This was clearly seen by a significant decrease $(P<0.05)$ in the serum of AST, ALT, ALP, and bilirubin as well as less pathological changes in liver sections compared with the $\mathrm{CCl}_{4}$-treated group.

The protection of $A$. digitata $\mathrm{L}$. fruit pulp against $\mathrm{CCl}_{4}$ induced liver damage and restoration of biochemical values could result from the fruit content of triterpenoids [65], $\beta$-sitosterol, $\beta$-amyrin palmitate or/and $\alpha$-amyrin, and ursolic acid along with the antioxidant, anti-inflammatory, analgesic, immunostimulant, and antimicrobial activities of A. digitata L. fruit pulp [66].

3.3. Argemone mexicana L., Papaveraceae, Khash Khash. The hepatoprotective activity of water and methanolic extracts of Argemone mexicana L. aerial part was evaluated against $\mathrm{CCl}_{4}$ damage in rats. The hepatic injury was achieved by injection of $\mathrm{CCl}_{4} 3 \mathrm{ml} / \mathrm{kg}$ s.c in olive oil $(1: 1 \mathrm{v} / \mathrm{v})$. The extracts were given at different doses, 100, 200, and $400 \mathrm{mg} / \mathrm{kg} /$ day for methanolic extract and $400 \mathrm{mg} / \mathrm{kg} /$ day orally for aqueous extract for 5 days. $\mathrm{CCl}_{4}$ was injected at the $3^{\text {rd }}$ day. The methanol extract at $100 \mathrm{mg} / \mathrm{kg}$ offered significant hepatoprotective activity $(P<0.05)$ by reducing serum ALT, AST, and ALP.

The results of the histopathology of methanol extract at a dose of $100 \mathrm{mg} / \mathrm{kg}$ showed healing of the liver parenchyma and regeneration of liver cells. The results were comparable with silymarin $(70 \mathrm{mg} / \mathrm{kg}$ orally). The mechanism by which $A$. mexicana displays hepatoprotection is not known yet [24].

3.4. Anogeissus leiocarpus (DC) Wall., Combretaceae, Sahib. Hepatoprotective effects of the Anogeissus leiocarpus bark ethanolic extract were investigated in rats against carbon 


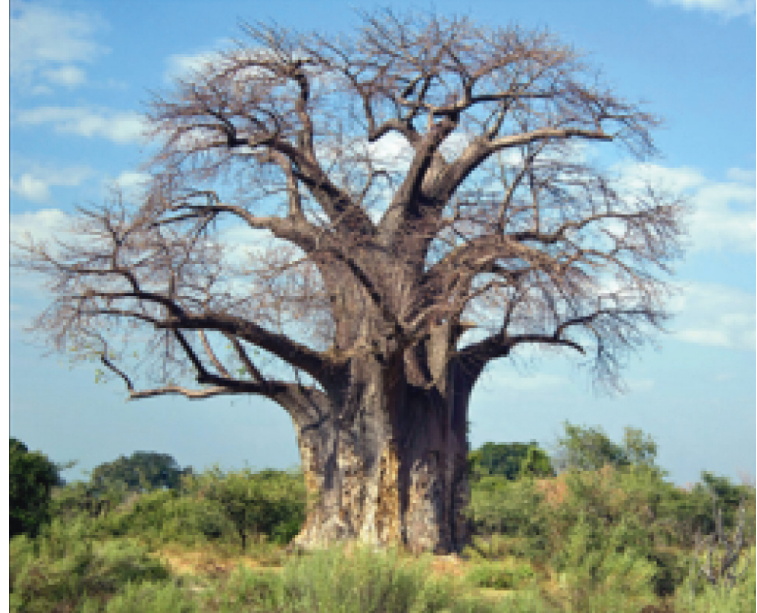

(a)

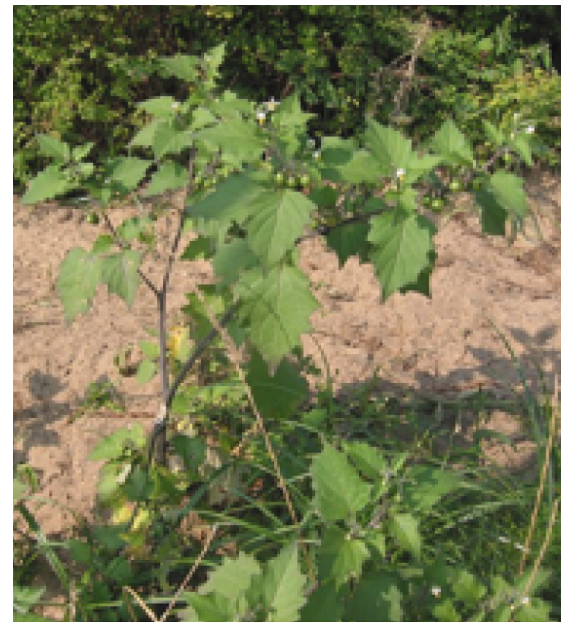

(d)

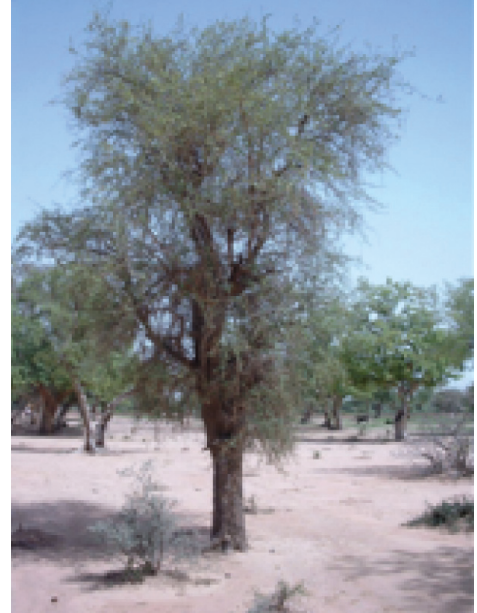

(b)

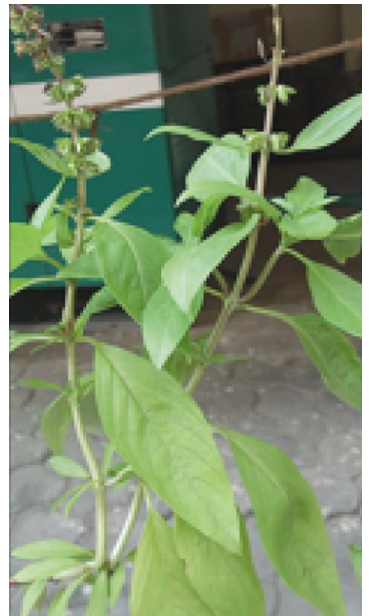

(c)

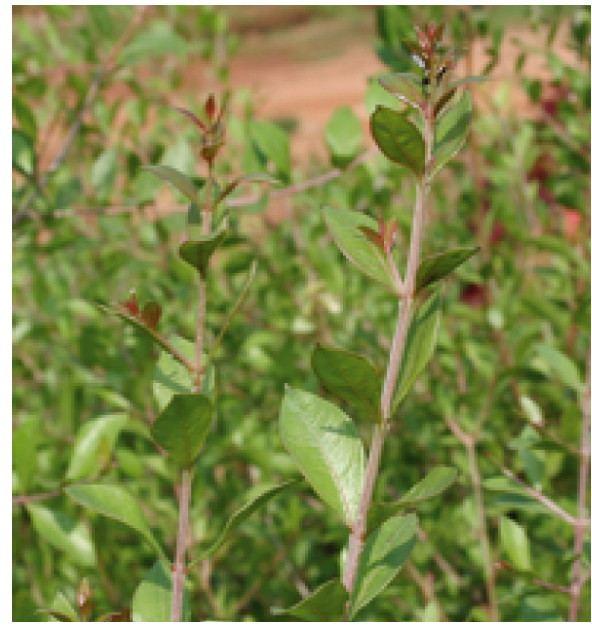

(e)

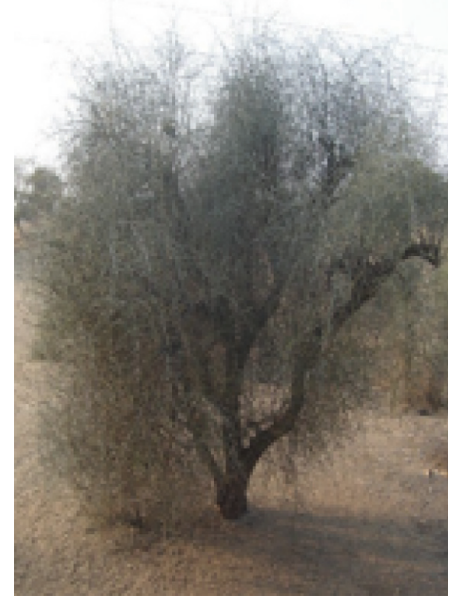

(f)

Figure 1: Some Sudanese medicinal plants (source: Wikimedia Commons): (a) Adansonia digitata, (b) Balanites aegyptiaca, (c) Occimum basilicum, (d) Solanum nigrum, (e) Lawsonia inermis, and (f) Capparis decidua.

tetrachloride- $\left(\mathrm{CCl}_{4^{-}}\right)$induced liver injury [40]. Rats received the plant extract three times at 0,12 , and 24 hours. $\mathrm{CCl}_{4}$ was injected $1.25 \mathrm{ml} / \mathrm{kg}$ as a single dose 30 minutes before the first dose of test extracts.

Blood samples were collected after $36 \mathrm{~h}$ for haematological and biochemical investigation before the rats were euthanized, and liver samples were taken for histopathology.

Phytochemical screening of the A. leiocarpus bark ethanolic extract indicated the presence of tannins, saponins, flavonoids, sterols, triterpenoids, and cumarins. Oral administration of the ethanolic extract of the plant at a dose of $200 \mathrm{mg} / \mathrm{kg}$ displayed a significant $(P<0.05)$ hepatorenal protective effect against $\mathrm{CCl}_{4}$ by lowering liver biomarkers (AST, ALT, and ALP), kidney biomarker levels (urea and creatinine), as well as normalizing haematological parameters when compared with standard drug silymarin. Histopathological investigation of liver and kidney tissues verified the protective effect of the plant extract.
The protective effect of the extract may be related mainly to the antioxidant property of its high content of flavonoids, tannins, sterols, and triterpenes [67-69].

3.5. Balanites aegyptiaca L., Balanitaceae, Hegleig Tree (Ar) Laloub Fruit. The hepatoprotective effects of water and methanolic extracts of Balanites aegyptiaca bark were investigated in rats injected with $0.2 \mathrm{ml} / \mathrm{kg}$ carbon tetrachloride $\left(\mathrm{CCl}_{4}\right)$ for 10 days. The extracts were administered at the same time with $\mathrm{CCl}_{4}$ at a dose of 250 to $500 \mathrm{mg} / \mathrm{kg}$ orally for 10 days. Significant decreases in AST, ALT, and ALP activities, bilirubin concentration, as well as mild hepatocyte lesions were noticed in rats administered with plant extracts compared with the $\mathrm{CCl}_{4}$ group [25].

Phytochemical screening of aqueous stem bark extracts of $B$. aegyptiaca indicated the presence of alkaloids, flavonoids, glycosides, phenols, saponins, and tannins [70]. 
Flavonoids, phenolic compounds, and saponins are known to have antioxidant and hepatoprotective effects. Furthermore, the flavonoids and saponins of B. aegyptiaca may stabilize reactive oxygen species by reacting with them and oxidizes subsequently to more stable and less reactive radicals $[70,71]$.

3.6. Cannabis sativa L., Cannabaceae, Bango, Hashish. The hepatoprotective activity of Cannabis sativa L. against $\mathrm{CCl}_{4}$-induced hepatotoxicity in rats was investigated [26]. Daily oral doses of C. sativa $\mathrm{L}$. oil 1 and $0.5 \mathrm{ml} / \mathrm{kg}$ body weight were administered to rats. The hepatotoxicity induced by injecting $\mathrm{CCl}_{4}$ in paraffin oil $(1: 9 \mathrm{v} / \mathrm{v})$ at a dose of $0.2 \mathrm{ml} / \mathrm{kg}$ for 10 days was found to be inhibited by simultaneous oral administration of $C$. sativa oil of at a dose of 1 and $0.5 \mathrm{ml} / \mathrm{kg}$. This was evidenced by the decreased levels of serum AST, ALT, ALP, and bilirubin. However, the liver changes were also inhibited compared with control rats and silymarin group. The oil of $C$. sativa contains a hepatoprotective ingredient that protects the liver from carbon tetrachloride damage [26].

3.7. Capparis decidua (Forsk.), Capparaceae, Tundub. The aqueous and methanolic extracts of Capparis decidua stems locally known as Altoundob were screened for their hepatoprotective activity against $\mathrm{CCl}_{4}$-induced hepatotoxicity in rats. The hepatotoxicity produced by administration of $\mathrm{CCl}_{4}$ in paraffin oil $(1: 9 \mathrm{v} / \mathrm{v})$ at a dose of $0.2 \mathrm{ml} \cdot \mathrm{kg}^{-1}$ for 10 days was found to be inhibited by simultaneous oral administration of aqueous and methanolic extracts of C. decidua stems (200, $400 \mathrm{mg} \cdot \mathrm{kg}^{-1}$ b.wt.) for 10 days, with evidence of decreased level of serum AST, ALT, ALP, and bilirubin. In addition, the concurrent administration of both extracts with $\mathrm{CCl}_{4}$ for 10 days masked the liver fatty changes induced by the hepatotoxic compound observed in the intoxicated control rats. The results were comparable with the hepatoprotective effect of the standard drug silymarin. The results showed that the aqueous extract of $C$. decidua had higher activity than the methanolic extract, probably related to more polar phytoconstituents [7, 27].

The preliminary phytochemical screening of the powdered plant showed the presence of alkaloids, flavonoids, tannins, sterols, saponins, cyanogenic glycosides, and cumarins as major constituents of the studied extracts.

The study revealed the evidence of the presence of flavonoids, cyanogenic glycosides and triterpenes which are antioxidants and may be responsible for the hepatoprotective property [13, 72-74].

In addition, $C$. decidua was found to alter superoxide dismutase and catalase enzyme levels to reduce oxidative stress. Moreover, the high contents, in the plant, of minerals such as iron and vitamins such as vitamin $\mathrm{C}$ may contribute to the extract hepatoprotectivity [75].

3.8. Combretum hartmannianum (Schweinf), Combretaceae, Habiel. Habeel Al Gabal. The hepatoprotective effect of Combretum hartmannianum leaves methanolic extract was investigated against $\mathrm{CC}_{4}{ }^{-}$and paracetamol-induced hepatotoxicity. The methanolic extract of C. hartmannianum $(12.5,25,50 \mathrm{mg} / \mathrm{kg})$ was injected intraperitoneally (IP) one hour before injection of $\mathrm{CCl}_{4}(800 \mathrm{mg} / \mathrm{kg} \mathrm{IP})$ and paracetamol ( $1 \mathrm{~g} / \mathrm{kg}$ P.O). The results were compared with silymarin, a standard hepatoprotective drug. C. hartmannianum leaves' extract showed hepatoprotective effect against $\mathrm{CC}_{4}$ induced hepatotoxicity which was evidenced by significant decrease in AST, ALT, ALP, and bilirubin and significant increase in serum total protein and albumin. Significant decreases in AST and ALT were also observed in rats given $C$. hartmannianum leveasthe methanolic extract of C. hartmannianum leaves IP one hour before oral administration of paracetamol to induce hepatotoxicity [28]. The methanolic extract of $C$. hartmannianum leaves contained flavonoids, tannins, saponins and unsaturated sterols and no cumarins, alkaloids, and triterpenoids [28]. The hepatoprotective effect of this plant may be due to high antioxidant activity in DPPH free radical scavenging assay and flavonoid content [76].

3.9. Dobera glabra (Forsk.), Salvadoraceae, Al Meikah. Dobera glabra leaves' aqueous and methanolic extracts were investigated for their hepatoprotective activity against $\mathrm{CCl}_{4}$ induced hepatotoxicity in rats. $\mathrm{CCl}_{4}$ was injected in paraffin oil $(1: 9 \mathrm{v} / \mathrm{v})$ at a dose of $0.2 \mathrm{ml} \cdot \mathrm{kg}^{-1}$ for 10 days. Leaf extracts were given by simultaneous oral administration at a dose of 200 and $400 \mathrm{mg} \cdot \mathrm{kg}^{-1}$ b.w. for 10 days. ALT, AST, and bilirubin were significantly elevated in plant extract groups compared with standard drug silymarin and $\mathrm{CC1}_{4}$ group. Moreover, administration of both extracts with $\mathrm{CCl}_{4}$ for 10 days produced diffuse centrilobuar hepatocyte necrotic lesions in hepatocytes [30].

The preliminary phytochernical screening of the powdered plant showed the presence of alkaloids, flavonoids, tannins, sterols, saponins, cyanogenic glycosides, and cumarins as major constituents of the studied extracts [30].

3.10. Khaya senegalensis (Desr.), Meliaceae, Mahogany Tree. The hepatoprotective activity of the aqueous extract of the bark of Khaya senegalensis was evaluated against $\mathrm{CCl}_{4}$-induced liver damage in rats. Bark extract was administered at a dose of 250 and $500 \mathrm{mg} / \mathrm{kg}$ for 5 days, while $\mathrm{CCl}_{4}(3 \mathrm{ml} / \mathrm{kg}$ body weight/rat) was injected subcutaneously on the $3^{\text {rd }}$ day of experiment. Silymarin $(50 \mathrm{mg} / \mathrm{kg})$ was also used as a standard drug. The levels of AST, ALT, ALP, and bilirubin in groups treated by the extract were significantly increased compared with that of the silymarin group; the hepatoprotective activity was supported by histopathological finding of liver tissue [30].

The hepatoprotective effect of the methanolic extract of the bark of Sudanese plant Khaya senegalensis, which is used in folk medicine for treatment of jaundice, was investigated. The hepatoprotective effect was tested in rats against $\mathrm{CCl}_{4}$ and paracetamol-induced hepatotoxicity. The extracts were given intraperitoneally one hour before injection of $\mathrm{CCl}_{4}$ $(800 \mathrm{mg} / \mathrm{kg}$ IP) and administration of paracetamol (1 and $2 \mathrm{~g} / \mathrm{kg}$ P.O). The results were compared with the standard 
hepatoprotective agent silymarin. The methanolic extract of the bark of $K$. senegalensis showed a hepatoprotective effects against $\mathrm{CCl}_{4}$ and paracetamol-induced hepatotoxicity, as evidenced by the significant decrease in ALT, AST and ALP. Moreover, dichloromethane and petroleum ether extracts against paracetamol-induced hepatotoxicity did not possess hepatoprotective effect as evidenced by failure to decrease ALT and AST activities. Chloroform and ethyl acetate extracts showed significant hepatoprotective effect against toxicity induced by paracetamol as evidenced by significant decrease of ALT and AST. The mild histopathological lesions observed in the groups treated with methanolic and chloroform extracts of the bark of $K$. senegalensis compared with paracetamol groups indicated some protective effect [32, 33].

Khaya senegalensis bark aqueous extract was also evaluated for its hepatoprotective activity against $\mathrm{CCl}_{4}$-induced liver damage in rats [31]. The bark of $K$. senegalensis was orally administered at a dose of 250 and $500 \mathrm{mg} / \mathrm{kg}$ for five days. The levels of AST, ALT, ALP, bilirubin, total protein, and albumin, in groups treated by the extract were significantly decreased compared with that of $\mathrm{CCl}_{4}$ group. These findings were strongly supported by the histopathological results of liver sections in comparison with the group treated by the standard drug silymarin. This study indicates that, the aqueous extract of $K$. senegalensis bark possesses hepatoprotective ingredients [31].

The hepatoprotective activity of $K$. senegalensis bark ethanolic extract was evaluated against $\mathrm{CCl}_{4}$ in rats [23]. The extract was given three times $(0,12$, and 24 hours $)$ at a dose of $200 \mathrm{mg} / \mathrm{kg}$ orally. $\mathrm{CCl}_{4}(1.25 \mathrm{ml} / \mathrm{kg})$ diluted in liquid paraffin $(1: 1)$ was injected subcutaneously 30 minutes after the administration of the first dose of vehicle as single dose. After $36 \mathrm{~h}$, blood samples were collected for biochemical and haematological investigations. The extract showed significant decrease in the level of serum enzymes ALT, AST, and ALP, as well as significant decrease in the concentration of total and direct bilirubin compared with the $\mathrm{CCl}_{4}$ group. The extract also masked $\mathrm{CCl}_{4}$-induced enlargement of the liver which confirmed the hepatoprotective effect of the plant extract.

The preliminary phytochemical screening of the powdered material of plants revealed the presence of tannins, sterols, saponins, cumarins, and triterpenes. The plant material was devoid of alkaloids, flavonoids, and anthraquinone compounds. The extract exerts weak antioxidant activity when tested by DPPH assay [23].

3.11. Kigelia africana (Lam.), Bignoniaceae, Umm Shutour. The hepatoprotective activity of aqueous and methanolic extracts of Kigelia africana was investigated against $\mathrm{CCl}_{4}$ induced liver damage on male Wistar rats. $\mathrm{CCl}_{4}$ was injected at a dose of $3 \mathrm{ml} / \mathrm{kg}$, subcutaneously (1:1 dilution with olive oil), on the $3^{\text {rd }}$ day of experiment. Silymarin was given orally $(50 \mathrm{mg} / \mathrm{kg} / \mathrm{day})$ for 5 days. Methanolic extract was given at a dose of 100,200 , and $400 \mathrm{mg} / \mathrm{kg} / \mathrm{day}$, while the aqueous extract was given at a dose of $400 \mathrm{mg} / \mathrm{kg} /$ day for 5 days. The study revealed that, administration of the two extracts (aqueous and methanol) of the plant seeds has toxic effects which resulted in alterations in haematological parameters (Hb, WBCs MCH, MCHC, and granulocytes) and also alterations in AST, ALT, and ALP activities. There were also histopathological alterations in the liver and kidney [34].

3.12. Lawsonia inermis L., Lythraceae, Henna. The hepatoprotective effect of Lawsonia inermis leaves' methanolic extract on $\mathrm{CCl}_{4}$-induced hepatotoxicity in rats was investigated. The leaves of $L$. inermis methanolic extract, which are obtained by maceration, were administered orally at a dose of $100 \mathrm{mg} / \mathrm{kg}$ and $200 \mathrm{mg} / \mathrm{kg}$. Silymarin $(25 \mathrm{mg} / \mathrm{kg})$, a potent hepatoprotective drug, was used as the standard control. The two doses of the plant extract showed dosedependent hepatoprotective effects, as evident by the significant reduction $(P<0.05)$ in serum levels of AST, ALT, ALP, and bilirubin along with the improvement in histopathological liver sections compared with $\mathrm{CCl}_{4}$-only treated animals. The results indicated that this plant material could provide a hepatoprotective effect that could be attributed to its antioxidant properties [35]. It has been reported that the plant extract affords hepatoprotective activity due to its antioxidant property attributed to the flavonoid contents that are effective scavengers of superoxide anions, peroxynitrite, peroxyl, and hydroxyl radicals [77].

3.13. Lepidium sativum L., Brassicaceae, Pepper Cress or ELRshad. The protective effect of Lepidium sativum L. seeds' methanolic extract (200 and $400 \mathrm{mg} / \mathrm{kg}$ ) was investigated against $\mathrm{CCl}_{4}$-induced liver damage in rats. $\mathrm{CCl}_{4}$ was injected simultaneously with the extracts at a dose of $0.2 \mathrm{ml} / \mathrm{kg}$ after dissolving in paraffin oil $(1: 9 \mathrm{v} / \mathrm{v})$ for 10 days. Mean serum AST, ALT, ALP, and bilirubin concentration was significantly reduced in rats given L. sativum extract. However, the severe fatty changes that shown in the liver sections of rats given $\mathrm{CCl}_{4}$ were significantly decreased in plant-treated groups. The mechanism of the hepatoprotective action of the plant is uncertain but may be due to inhibition of lipid peroxidation in the liver.

The presence of flavonoids, triterpens, alkaloids, tannins, and coumarins in L. sativum explains its role in hepatoprotection by inhibiting the free radical-mediated damage. In addition, flavonoids, triterpenes, and tannins were antioxidant agents and interfere with free radical formation [36].

3.14. Moringa oleifera Lam., Moringiaceae, Al-rawag. Hepatoprotective activity of Moringa oleifera leaves aqueous extract was investigated against $\mathrm{CCl}_{4}$-induced liver injury in rats. Rats received $\mathrm{CCl}_{4}$ in paraffin oil $(1: 9 \mathrm{v} / \mathrm{v})$ at a dose of $0.2 \mathrm{ml} \cdot \mathrm{kg}^{-1}$ for 10 days to induce hepatocellular damage. M. oleifera extract was given concurrently with $\mathrm{CCl}_{4}$ at a dose of 200 and $400 \mathrm{mg} \cdot \mathrm{kg}^{-1}$; p.o. The simultaneous administration of the aqueous extract with $\mathrm{CCl}_{4}$ for 10 days reduced the levels of serum AST, ALT, ALP, and bilirubin. Moreover, the liver fatty change observed in the intoxicated control rats was also reduced especially at a dose of $200 \mathrm{mg} \cdot \mathrm{kg}^{-1}$. Degenerative changes with cytoplasmic 
rarefication and acidophilic cytoplasm with pycknotic nuclei were observed precentrally in the livers of rats that received $M$. oleifera leaves' aqueous extract at a dose rate of $400 \mathrm{mg} \cdot \mathrm{kg}^{-1}$. The results of this study indicated that the aqueous extract of $M$. oleifera leaves could afford significant protection against $\mathrm{CCl}_{4}$-induced liver injury in rats especially at lower dose [37]. Furthermore, the preliminary phytochemical study revealed the presence of alkaloids, saponins, flavonoids, tannins, sterols, glycosides, and cumarins [37]. It has been reported that a number of phytoconstituents have protective effects on liver due to antioxidant properties such as flavonoids, triterpenoids, and sterols. Presence of those compounds in $M$. oleifera may be responsible for the protective effect on $\mathrm{CCl}_{4}$-induced liver damage in rats $[12,78]$. Also, a number of scientific studies reported that $M$. oleifera is known to be a source of antioxidants due to its total phenolic, vitamin $\mathrm{A}$, and vitamin $\mathrm{E}$ contents which is known to reduce lipid peroxidation $[79,80]$.

3.15. Nigella sativa L., Ranunculaceae, Black Seed, Black Cumin, or Habat Elbarka. The protective effects of Nigella sativa seed extract (NSSE) against acetaminophen- (APAP-) induced hepatotoxicity in TIB-73 cells and rats were investigated. Toxicity in TIB-73 cells was induced with $10 \mathrm{mmol} / \mathrm{L}$ APAP, and the protective effects of NSSE were evaluated at $25,50,75$, and $100 \mathrm{mg} / \mathrm{mL}$. For in vivo examination, a total of 30 rats were equally divided into five experimental groups: normal control (vehicle), APAP ( $800 \mathrm{mg} / \mathrm{kg}$ body weight single IP injection) as a hepatotoxic control, and three APAP and NS pretreated (2 weeks) groups (APAP + NSSE $100 \mathrm{mg} ; \quad$ APAP + NSSE $300 \mathrm{mg}$; and APAP + NSSE $900 \mathrm{mg} / \mathrm{kg})$. TIB-73 cell viability was drastically decreased by $(49.0 \pm 1.9) \%$ after $10 \mathrm{mmol} / \mathrm{LAPAP}$ treatment, which also increased reactive oxygen species production. Cotreatment with NSSE at 25, 50, 75, and $100 \mathrm{mg} / \mathrm{mL}$ significantly improved cell viability and suppressed reactive oxygen species generation. In vivo, the APAP induced alterations in blood lactate levels, $\mathrm{pH}$, anionic gap, and ion levels ( $\mathrm{HCO} 3-, \mathrm{Mg} 2+$, and $\mathrm{K}+$ ), which tended to normalize with the NSSE pretreatment. The NSSE also significantly decreased serum ALT, AST, and ALP, which correlated with decreased levels of hepatic lipid peroxidation (malondialdehyde), increased superoxide dismutase levels, and reduced glutathione concentrations. Improved hepatic histology was also found in the treatment groups other than the APAP group [39]. The protective effects of $N$. sativa seed extract against APAP-induced hepatotoxicity and metabolic disturbances in rats might be related to improved antioxidant activity and attenuation of oxidative stress, lipid peroxidation, and ROS generation.

Aqueous extract of $N$. sativa seeds was tested for its hepatoprotective effect against $\mathrm{CCl}_{4}$-induced hepatotoxicity in rats. Plant extract was given at a dose of 250 and $500 \mathrm{mg}$ / $\mathrm{kg}$ for 5 days. $\mathrm{CCl}_{4}$ was injected $(3 \mathrm{ml} / \mathrm{kg})$ subcutaneously on the $3^{\text {rd }}$ day. Silymarin $(50 \mathrm{mg} / \mathrm{kg})$ was used as a reference hepatoprotective drug. Rats were scarified after 5 days. The levels of serum AST, ALT, ALP, and total protein (TP) were significantly decreased in groups treated with extract (250 and $500 \mathrm{mg} / \mathrm{kg}$ ) compared with that of $\mathrm{CCl}_{4}$. The biochemical observations were correlated with histopathological examination of rat liver sections [30].

In another study, $N$. sativa methanolic seeds' extract was investigated in $\mathrm{CCl}_{4}$-induced liver damage in rats. Rats were IP administered with $\mathrm{CCl}_{4}\left(0.2 \mathrm{ml} \cdot \mathrm{kg}^{-1}\right.$ dissolved in liquid paraffin $1: 9)$ together with 250 and $500 \mathrm{mg} \cdot \mathrm{kg}^{-1}$ of the methanolic extract of $N$. sativa which was dissolved in dimethylsulfoxide. Rats were scarified after 10 days. The levels of ALT, AST, and ALP were found to be significantly higher in both $\mathrm{CCl}_{4}$ - and $N$. sativa-treated groups compared with the controls, but the increase was less in the groups which were treated with $N$. sativa methanolic extract. The histopathological changes in the livers of the $\mathrm{CCl}_{4}$ group exhibited severe centrilobular vacuolation and congestion but in the groups treated with 250 and $500 \mathrm{mg} \cdot \mathrm{kg}^{-1} \mathrm{~b}$. wt., these changes were to a lesser extent [38].

3.16. Ocimum basilicum L., Lamiaceae, Alryhan. The hepatoprotective activity of Ocimum basilicum L. whole plant ethanolic extract was evaluated. The extract was tested on rats at an oral dose of $200 \mathrm{mg} / \mathrm{kg}$ for hepatoprotective effect before injection of $\mathrm{CCl}_{4}(0 \mathrm{~h})$ and following posttreatment with $\mathrm{CCl}_{4}$ at 12 and 24 hours. Rats received the $\mathrm{O}$. basilicum extract three times at 0,12 , and 24 hours, while the $\mathrm{CCl}_{4}$ was injected once at a dose of $1.25 \mathrm{ml} / \mathrm{kg} 30$ minutes before the first dose of test extracts.

Blood samples were collected after $36 \mathrm{~h}$ for haematological and biochemical investigation before the rats were euthanized, and liver samples were taken for histopathology.

O. basilicum ethanol extract at a dose of $200 \mathrm{mg} / \mathrm{kg}$ exhibited a significant $(P<0.05)$ protective effect by lowering serum levels of AST, ALT, and ALP comparable with that of silymarin used as a standard drug compared with $\mathrm{CCl}_{4}$. Haematological parameters were also found similar to the silymarin group. These biochemical observations were supplemented by histopathological examination of liver which proved to be protected by the plant extract. The study concluded that $O$. basilicum ethanolic extract has a potential hepatoprotective activity against $\mathrm{CCl}_{4}$-induced hepatotoxicity in rats [40].

Phytoconstituents identified in O. basilicum whole plant ethanolic extract included flavonoids, alkaloids, tannins, saponins, triterpenes, sterols, and cumarins.

The protective effect of the extract probably related to the antioxidant property due to its high content of flavonoids, saponin, tannins, sterols, and triterpenes and due to its superoxide radical and nitric oxide radical scavenging activities [81-83].

3.17. Phoenix dactylifera Linn., Palmae Date, Date Palm. The hepatoprotective effect of Sudanese dates palm (Phoenix dactylifera Linn) pollen grain powder (produced from Elgolid Locality, Northern State of Sudan) in albino rats was evaluated. The hepatotoxicity was achieved by injecting $\mathrm{CCl}_{4}$ subcutaneously $(3 \mathrm{ml} / \mathrm{kg}$ dissolved in equal preparation with olive oil $1: 1$ ) on the $3^{\text {rd }}$ day. Date palm pollen grains were 
given orally at a dose of 250 and $500 \mathrm{mg} / \mathrm{kg}$ for five days. Silymarin was given orally $(50 \mathrm{mg} / \mathrm{kg}$ ) as a reference drug for 5 days. Date palm pollen grain powder showed significant $(P<0.05)$ hepatoprotective activity which was indicated by decreased of ALT, AST, and ALP levels of treated groups compared with $\mathrm{CCl}_{4}$. These results correlated with histopathological changes which proved centrilobular necrosis with slight congestion [41].

The mechanism by which the date palm fruit extract induced its hepatoprotective activity is not certain. However, it could be due to $\beta$-sitosterol, a constituent of $P$. dactylifera L. which may be partly responsible for the protective activity against hepatotoxicity [84]. It is suggested that flavonoids in $P$. dactylifera L. could be contributing as a factor to its ability for hepatoprotection through inhibition of cytochrome P-450 aromatase [85]. In addition, the high content of vitamin $\mathrm{C}$ in the date palm pollen grains may also play a role in hepatoprotection [41].

3.18. Raphanus sativus L., Cruciferae, Alfgel. Raphanus sativus L. water and methanolic extracts were investigated for a possible hepatoprotective activity against carbon tetrachloride-induced hepatotoxicity in rats. $\mathrm{CCl}_{4}(0.2 \mathrm{ml} / \mathrm{kg}$ in paraffin oil 1:9 v/v) was administered IP with either methanolic or water extract at doses of 200 and $400 \mathrm{mg} \cdot \mathrm{kg}^{-1}$. The animals were sacrificed after 10 days. Biochemical results showed that $\mathrm{CC}_{4}$ induced hepatotoxicity which was reduced by the use of the plant as indicated by inhibition of the increased serum AST, ALT, and ALP activities and bilirubin concentration besides histopathological changes. The phytochemical tests revealed presence of triterpenes, alkaloids, flavanoids, tannins, saponin, and cournarins but negative for cyanogenic glycosides and anthraquinone glycosides [42].

3.19. Solanum nigrum L., Solanaceae, Enab Eldib. The hepatoprotective effects of Solanum nigrum water and methanolic extracts were investigated in rats injected with $0.2 \mathrm{ml} /$ $\mathrm{kg} \mathrm{CCl}_{4}$ for 10 consecutive days. S. nigrum extracts were administered orally at a dose of 250 to $500 \mathrm{mg} / \mathrm{kg}$ for 10 days. The extracts showed a hepatoprotective effect against $\mathrm{CCl}_{4^{-}}$ induced liver damage, which was evident by the decrease in serum AST, ALT, and ALP activities and bilirubin concentration and by mild histopathological lesions when compared with the group of rats injected with $\mathrm{CCl}_{4}$ alone. The water extract appears to have a better hepatoprotective effect than the methanolic one which could be due to more polar phytoconstituents [43].

3.20. Sterculia setigera Del., Sterculiaceae, Terter. The ethanolic and ethyl acetate extracts of Sterculia Setigera stem bark that belongs to the family of Sterculiaceae was evaluated for its hepatoprotective activity in albino rats with liver damage induced by carbon tetrachloride $\left(\mathrm{CCl}_{4}\right)$. The hepatotoxicity was produced by the administration of $\mathrm{CCl}_{4}$ at a dose of $0.2 \mathrm{ml} / \mathrm{kg}$ for 10 days. The extracts of $S$. Setigera stem bark were given orally at a dose of 200 and $400 \mathrm{mg} / \mathrm{kg}$ body weight. The extracts exhibited moderate protective effect by lowering the serum levels of ALT, AST, ALP, total protein, albumin, and bilirubin concentration to significant extent. In addition, the concurrent administration of the plant extracts with $\mathrm{CCl}_{4}$ for 10 days masked the liver changes induced by the hepatotoxic compound in rats and compared with hepatoprotective effect of the standard drug Silymarin. However, severe necrotic hepatic lesions induced by $\mathrm{CCl}_{4}$ were reduced by ethanolic and ethyl acetate extract of the plant [44].

3.21. Tamarindus indica L., Caesalpinaceae, Aradaib. The fruit pulp ethanolic extract of Tamarindus indica was investigating in protecting liver damage induced by $\mathrm{CCl}_{4}$. Ethanolic extract of $T$. indica was administered at a dose of $150 \mathrm{mg} / \mathrm{kg} /$ day for 5 days. Silymarin $(50 \mathrm{mg} / \mathrm{kg}$ ) was used as a standard drug. $\mathrm{CCl}_{4}$ was injected subcutaneously at $0.2 \mathrm{ml} /$ $\mathrm{kg}$ diluted $(1: 9)$ in liquid paraffin on day 2 and 3 and received saline orally for 5 days. Results indicated that, the ethanolic extract of $T$. indica ameliorated the damage caused by $\mathrm{CCl}_{4}$ by lowering the levels of AST, ALT, and ALP and the concentration of bilirubin, and this effect was verified by improvement of histopathological picture in the livers of the group treated by the plant compared with the liver of the $\mathrm{CCl}_{4}$ group which exhibited severe centrilobular necrosis and fatty vacuolation. The study indicates that $T$. indica ethanolic extract possess hepatoprotective ingredients [45].

The presence of moderated concentration of tannins and alkaloids and low concentration of flavonoids and saponin in the ethanolic extract of the fruit pulp of $T$. indica could be responsible for the membrane-stabilizing activity [86].

\section{Discussion}

In Sudan, medicinal plants possess a distinctive position in folk medicine as well as their important position in the sociocultural and spiritual arena of rural and tribal life in Sudan. Throughout Sudan's long history, local indigenous cultures have merged with many other cultures such as Pharaonic, Christian, and Islamic. This has helped traditional medicine and the use of medicinal plants to become an important part of Sudan's cultural heritage [87].

In Sudan, people in rural areas prefer treatment of various diseases by medicinal plants than by modern synthetic drugs which are expensive and because of difficulty to access medical services particularly in areas of conflicts and political instability [88]. This review presents data on 21 Sudanese herbs evaluated scientifically as hepatoprotective using well-known experimental models. From this review, it is clear that the medicinal plants play an important role in the treatment of a variety conditions including liver diseases. Most medicinal plants are widely distributed in the rural areas. Carbon tetrachloride and paracetamol as hepatotoxins are used widely to assess hepatoprotective plants in Sudan.

The hepatoprotective activity of these plants is based on lowering liver biomarkers as well as suppressing of degenerative changes in hepatocytes produced by the hepatotoxic agent compared with reference hepatoprotective 
drug (silymarin). The evaluation is usually based on administration of different doses of plant extracts using aqueous or chemical extracts such as methanol, ethanol, petroleum ether, and ethyl acetate.

The majority of reports used either methanol or aqueous extracts (alone or with other solvents), with the exception of eight studies in which both extracts were evaluated by similar protocols and dosages (C. decidua, B. aegyptiaca, A. Mexicana, S. nigrum, M. oleifera, $R$. sativus, D. glabra, and $K$. africana). In positive plants ( $C$. decidua, B. aegyptiaca, $A$. Mexicana, S. nigrum, $M$. oleifera, and $R$. sativus), the studies investigated the aqueous and methanolic extracts of $C$. decidua, S. nigrum, and $M$. oleifera and clearly concluded that the aqueous extracts have better activity compared with methanolic one especially in lower doses [7, 27, 37, 43]. In contract, the study by [24] indicated that the hepatoprotective effect of $A$. mexicana is more pronounced in low dose $(100 \mathrm{mg} / \mathrm{kg})$ of methanolic extract than the aqueous extract. But in much higher dosage $(400 \mathrm{mg} / \mathrm{kg})$, the aqueous extract was more effective.

The activity of $R$. sativus and B. aegyptiaca in both extracts seem to be comparable and the higher dosages are the best $[25,42]$. Two studies evaluated hepatoprotective activity of aqueous and methanolic extracts of the leaves of Dobera glabra and the seeds of Kigelia Africana. Both plant extracts were not active at the dosage used, and rather seemed to have hepatotoxic effects $[29,34]$. The biochemical parameters used to verify the activity of both plant extracts did not differ statistically from the $\mathrm{CCl}_{4}$ group, and the severe necrotic hepatic lesions induced by $\mathrm{CCl}_{4}$ were not reduced to significant level by the administration of the extracts.

The mechanisms of action as hepatoprotective might be due to antioxidant properties of these plants which have major role in protecting liver cells from oxidative stress. Moreover, available information on this review clearly showed that these herbs are richest of phytoconstituents such as coumarins, terpenoids, glycosides, flavonoids, alkaloids, saponins, and tannins which are well known as hepatoprotective agents [27].

One of the noteworthy weaknesses in the design of most of the cited studies is that the methods used to test the hepatoprotective properties of these plants that most of the studies used cotreatment and pretreatment regimens (meaning that the plant extracts were given either before, or with, $\mathrm{CCl}_{4}$ and paracetamol). This is problematic for two reasons: First, both $\mathrm{CCl}_{4}$ and paracetamol toxicity depend upon conversion to reactive metabolites by cytochromes P450. Obviously, pretreating or cotreating with a milieu of phytochemicals has great potential to interfere in that metabolism [19]. Thus, such experiments may not actually demonstrate that the plants are hepatoprotective, but simply that they compete for drug-metabolizing enzymes. Second, pretreatments are not clinically relevant. A disease cannot be treated before it develops.

Further investigation should be done to evaluate the posttreatment efficacy of these plants using animal experimental models against $\mathrm{CCl}_{4^{-}}$and paracetamol-induced liver damage. Future studies also should elucidate the exact mechanisms of actions of the active plants. Clinical trials have to be conducted to evaluate efficacy, safety, proper dosage, and pharmacokinetic aspects of the active plant extracts in order to develop safe and effective dosage forms from these plants.

\section{Conclusion}

This review shows that some Sudanese medicinal plants have a crucial role in protecting liver from chemical injuries using in vivo and in vitro models. Herbal drugs in Sudan play a vital role in the primary health care since $90 \%$ of Sudanese people use medicinal plants to treat different diseases including liver disorders [11], due to high cost and inadequacy of conventional drugs used in the treatment of liver diseases. The action of these plants may be due to phytoconstituents, antioxidant, and anti-inflammatory effects. Further studies on these plants are necessary to establish the efficacy, safety, and exact mechanism of action as a moral alternative in the treatment of liver disorders.

Advanced tools and equipment are needed for identification, isolation, and purification of the active ingredients of the hepatoprotective plants and to examine their efficacy and safety through controlled clinical trials. There should be a motivation plan to Medicinal Plants Research Centre in Sudan to create cooperative collaborative research activities among the involved institutions.

\section{Conflicts of Interest}

The authors declare that they have no conflicts of interest.

\section{Authors' Contributions}

Ali S. A contributed to data acquisition and scientific writing. Sharief' N. H contributed to data collection. Mohamed Y. S provided the plant images and responsible for critical revision of the plant names and family, distribution in Sudan, and their traditional uses.

\section{Acknowledgments}

The authors are grateful to Prof. Ahmed Abdel-Rahim Gameel University of Khartoum, Department of Pathology, for his valuable help and encouragement of this review.

\section{References}

[1] G. E. B. ELGhazali, M. S. Eltohami, and A. A. B. Elegami, Medicinal Plants of the Sudan, Part III Medicinal Plants of White Nile Province, National Centre for Research. Khartoum University Press, Khartoum, Sudan, 1994.

[2] G. E. B. ELGhazali, Aromatic Plants of the Sudan, National Centre for Research. Khartoum University Press, Khartoum, Sudan, 2004.

[3] C.-H. Lee, S.-W. Park, Y. S. Kim et al., "Protective mechanism of glycyrrhizin on acute liver injury induced by carbon tetrachloride in mice," Biological \& Pharmaceutical Bulletin, vol. 30, no. 10, pp. 1898-1904, 2007.

[4] M. R. Ahsan, K. M. Islam, I. J. Bulbul, M. A. Musaddik, and E. Haque, "Hepatoprotective activity of methanol extract of some medicinal plants against carbon tetrachloride-induced 
hepatotoxicity in rats," European Journal of Scientific Research, vol. 37, no. 2, pp. 302-310, 2009.

[5] X. Shen, Y. Tang, R. Yang, L. Yu, T. Fang, and J.-a. Duan, "The protective effect of Zizyphus jujube fruit on carbon tetrachloride-induced hepatic injury in mice by anti-oxidative activities," Journal of Ethnopharmacology, vol. 122, no. 3, pp. 555-560, 2009.

[6] C. Girish, B. C Koner, S Jayanthi, K. R Rao, B Rajesh, and S. C Pradhan, "Hepatoprotective activity of six polyherbal formulations in paracetamol induced liver toxicity in mice," Indian Journal of Medical Research, vol. 129, no. 5, pp. 569578, 2009.

[7] S. A. Ali, T. H. Al-Amin, A. H. Mohamed, and A. A. Gameel, "Hepatoprotective activity of aqueous and methanolic extracts of Capparis decidua stems against carbon tetrachloride induced liver damage in rats," Journal of Pharmacology and Toxicology, vol. 4, no. 4, pp. 167-172, 2009.

[8] S. Handa, A. Sharma, and K. K. Chakraborti, "Natural products and plants as liver protecting drugs," Fitoterapia, vol. 57, no. 5, pp. 307-351, 1986.

[9] S. Pradhan and C. Girish, "Hepatoprotective herbal drug, silymarin from experimental pharmacology to clinical medicine," Indian Journal of Medical Research, vol. 124, no. 5, pp. 491-504, 2006.

[10] D. S. Raj, C. Aiyavu, J. J. Vennila, and K. Panneerselvam, "The hepatoprotective effect of alcoholic extract of Annona squamosa leaves on experimentally induced liver injury in Swiss albino mice," International Journal of Integrative Biology, vol. 5, no. 3, p. 182, 2009.

[11] UNIDO, Project for the Government of Sudan Prepared by UNIDO Industrial Utilization of Medicinal and Aromatic Plants in Sudan, UNIDO, Vienna, Austria, 1996.

[12] M. Gupta, U. K. Mazumder, T. S. Kumar, P. Gomathi, and R. S. Kumar, "Antioxidant and hepatoprotective effects of Bauhinia racemosa against paracetamol and carbon tetrachloride induced liver damage in rats," Iranian Journal of Pharmacology and Therapeutics, vol. 3, no. 1, pp. 12-20, 2004.

[13] W. Evans, Trease and Evans: Pharmacognocy. Fifteenth International Edition, WB Saunders, Philadelphia, PA, USA, 2002.

[14] A. N. B. Singab, D. T. A. Youssef, E. Noaman, and S. Kotb, "Hepatoprotective effect of flavonol glycosides rich fraction from egyptianVicia calcarata desf. Against CCI4-induced liver damage in rats," Archives of Pharmacal Research, vol. 28, no. 7, pp. 791-798, 2005.

[15] H. M. ELAmin, Trees \& Shrubs of the Sudan, Itaca Press, Exeter, UK, 1990.

[16] G. B. E. El-Ghazali, S. M. El-Tohami, and E.-E. Awatif, "Medicinal plants of the Sudan," in Medicinal Plants of Northern Kordofan, Omdurman Islamic University Printing and Publishing House, Omdurman, Sudan, 1997.

[17] L. W. D. Weber, M. Boll, and A. Stampfl, "Hepatotoxicity and mechanism of action of haloalkanes: carbon tetrachloride as a toxicological model," Critical Reviews in Toxicology, vol. 33, no. 2, pp. 105-136, 2003.

[18] D. Scholten, J. Trebicka, C. Liedtke, and R. Weiskirchen, "The carbon tetrachloride model in mice," Laboratory Animals, vol. 49, no. 1_suppl, pp. 4-11, 2015.

[19] H. Jaeschke, M. R. McGill, C. D. Williams, and A. Ramachandran, "Current issues with acetaminophen hepatotoxicity-a clinically relevant model to test the efficacy of natural products," Life Sciences, vol. 88, no. 17-18, pp. 737-745, 2011.
[20] S. J. Polyak, C. Morishima, V. Lohmann et al., "Identification of hepatoprotective flavonolignans from silymarin," Proceedings of the National Academy of Sciences, vol. 107, no. 13, pp. 5995-5999, 2010.

[21] A. H. Arbab, M. K. Parvez, M. S. Al-Dosari et al., "Hepatoprotective and antiviral efficacy of Acacia mellifera leaves fractions against hepatitis B virus," BioMed Research International, vol. 2015, Article ID 929131, 10 pages, 2015.

[22] M. A. Mohamed, I. A. A. Mohamed, T. H. Tag El-Din, A. Hassan, and M. Hassan, "Hepatoprotective effect of Adansonia digitata L. (Baobab) fruits pulp extract on $\mathrm{CCl}_{4^{-}}$ induced hepatotoxicity in rats," World Journal of Pharmaceutical Research, vol. 4, no. 8, pp. 368-377, 2015.

[23] B. A. Ahmed, Hepatoprotective, antioxidant and antibacterial effects of Ocimum basilicum, Anogeissus leiocarpus, Khaya senegalensis and Capparis decidua against $\mathrm{CCl}_{4}$ induced hepatotoxicity in rats, $\mathrm{PhD}$. thesis, Sudan Academy of Sciences (SAS), Council of Bioscience, Advanced Technology and Environment, Sudan, 2015.

[24] S. Adam, I. A. Idris, E. H. Abdelgadir, R. H. Ahmed, and E. E. Kamal, "Evaluation of hepatoprotective activity of Argemone mexicana aerial Part Extracts on CCL_4 induced liver damage in wistar rats," British Journal of Pharmacology and Toxicology, vol. 2, no. 5, pp. 251-256, 2011.

[25] R. A. M. Elhag, "Hepatoproteative activity of two Sudanese plants on experimental liver damage," M.Sc. thesis, Faculty of Veterinary Science, Sudan, 2001.

[26] E. Musa, E. L. Badwi, E. A. Osman, and M. M. Dahab, "Hepatoprotective and toxicity assessment of Cannabis sativa seed oil in Albino rat," International Journal of Clinical and Biological Sciences, vol. 1, pp. 69-76, 2012.

[27] S. A. Ali, A. A. Gameel, A. H. Mohamed, and T. Hassan, "Hepatoprotective activity of Capparis decidua aqueous and methanolic stems extracts against carbon tetrachloride induced liver histological damage in rats," Journal of Pharmacology and Toxicology, vol. 6, no. 1, pp. 62-68, 2011.

[28] H. M. E. Mohammed, "Pharmacological studies of selected plants with putative hepatoprotective activities," Thesis, Faculty of Pharmacy, Khartoum University, Khartoum, Sudan, 2008.

[29] S. A. Ali, "Hepatoproteative activity of selected sudanese plants on murine induced liver damage," MSc. thesis, Faculty of Veterinary Science, Sudan, 2005.

[30] S. A. M. Ali, "Hepatoprotective activity of aqueous extracts of Nigella sativa seeds and Khaya senegalensis bark in rats," Thesis, Faculty of Veterinary Medicine. Khartoum University, Khartoum, Sudan, 2011.

[31] S. Ali, S. M. A. Elbadwi, T. M. Idris, and K. M. Osman, "Hepatoprotective activity of aqueous extract of Khaya senegalensis bark in rats," Journal of Medicinal Plants Research, vol. 5, no. 24, pp. 5863-5866, 2011.

[32] H. M. Elagib, S. A. Shadad, A. E. Muddathir, Y. O. Mohammed, and S. M. Elagib, "Hepatoprotective activity of the methanolic extract of the bark of Khaya senegalensis in rats against carbon tetrachloride $\left(\mathrm{CCl}_{4}\right)$-induced hepatotoxicity in adose of $(800 \mathrm{mg} / \mathrm{kg}$ I.P)," International Journal of Science and Research, vol. 3, no. 3, pp. 1-9, 2014.

[33] H. M. Elagib, S. A. Shadad, A. E. Muddathir, Y. O. Mohammed, and S. M. Elagib, "Hepatoprotective activity of the methanolic extract of the bark of Khaya senegalensis in rats against paracetamol-induced hepatotoxicity," International Journal of Science and Research, vol. 3, no. 3, pp. 127-134, 2014.

[34] S. Shama, I. Y. Adam, M. Marwa, and I. A. Alhameed, "Kigelia africana fruits' extracts anti hepato-toxic effects on male 
wistar rats liver destruction induced by $\mathrm{CCL}_{4}$," Asian Journal of Medical Sciences, vol. 5, no. 1, pp. 26-32, 2013.

[35] M. Mohamed, I. Eldin, A. Mohammed, and H. Hassan, "Effects of Lawsonia inermis L. (Henna) leaves' methanolic extract on $\mathrm{CCl}_{4}$-induced hepatotoxicity in rats," Journal of Intercultural Ethnopharmacology, vol. 5, no. 1, p. 22, 2016.

[36] A. I. Abuelgasim, H. Nuha, and A. Mohammed, "Hepatoprotective effect of Lepidium sativum against carbon tetrachloride induced damage in rats," Research Journal of Animal \& Veterinary Sciences, vol. 3, pp. 20-23, 2008.

[37] S. Ali, A. H. Mohamed, A. A. Gameel, and T. Hassan, Protective Effect of Moringa Oleifera Leaves Aqueous Extract against Carbon Tetrachloride Induced Hepatotoxicity in Rats, Science Congress Faculty of Veterinary Medicine, Assiut, Egypt, 2010.

[38] A. I. Abuelgasim, E. A. Omer, and B. Elmahdi, "The effectiveness of Nigella sativa against liver damage in rats," Research Journal of Medicinal Plants, vol. 2, no. 1, pp. 43-47, 2008.

[39] G. O. Adam, M. M. Rahman, S.-J. Lee et al., "Hepatoprotective effects of Nigella sativa seed extract against acetaminopheninduced oxidative stress," Asian Pacific Journal of Tropical Medicine, vol. 9, no. 3, pp. 221-227, 2016.

[40] B. A. Ahmed, A. H. Mohamed, S. A. Ali, and A. I. Abuelgasim, "Hepatoprotective activity of ethanol extract of Ocimmum basillicum against $\mathrm{CCl}_{4}$-induced hepatotoxicity in albino rats," Journal of Natural and Medical Sciences, vol. 16, no. 2, pp. 11-18, 2015.

[41] I. A. A. Abuowf and A. Abuowf, Hepatoprotective Activity of Date Palm (Phoenix dactylifera) Pollen Grains in Rats, University of Khartoum, Khartoum, Sudan, 2009.

[42] N. H. S. H. Mohammed, A. I. Abelgasim, and A. H. Mohammed, "Protective effect of Raphanus sativus against carbon tetrachloride induced hepatotoxicity in wistar albino rats," Journal of Pharmacology and Toxicology, vol. 3, no. 4, pp. 272-278, 2008.

[43] R. A. M. Elhag and S. M. A. ElBadwi, "Hepatoprotective activity of Solanum nigrum extracts on chemically induced liver damage in rats," Journal of Veterinary Medicine and Animal Health, vol. 3, no. 4, pp. 45-50, 2011.

[44] T. H. Abdelrahman, "Hepatoprotective activity of ethanolic and ethyl acetate extracts of Sterculia Setigera against carbon tetrachloride induced hepatotoxicity in albino rats," Mediterranean Journal of Biosciences, vol. 1, no. 3, pp. 114-119, 2016.

[45] S. M. A. El Badwi, N. M. El Bagir, and A. E. Amin, "Protective effect of ethanolic extract of Tamarindus indica against $\mathrm{CCl}_{4}$ induced liver damage in rats," Australian Journal of Basic and Applied Sciences, vol. 7, no. 2, pp. 813-818, 2013.

[46] A. Koch, P. Tamez, J. Pezzuto, and D. Soejarto, "Evaluation of plants used for antimalarial treatment by the Maasai of Kenya," Journal of Ethnopharmacology, vol. 101, no. 1-3, pp. 95-99, 2005.

[47] D. Kaboré, S.-L. Hagrétou, D. Bréhima, C. Clarise, H. D. Mamoudou, and J. Mogens, "A review of baobab (Adansonia digitata) products: effect of processing techniques, medicinal properties and uses," African Journal of Food Science, vol. 5, no. 16, pp. 833-844, 2011.

[48] C. L. Priya and K. V. B. Rao, "Ethanobotanical and current ethanopharmacological aspects of Argemone mexicana linn: an overview," International Journal of Pharmaceutical Sciences and Research, vol. 3, no. 7, p. 2143, 2012.

[49] T. S. Sourabié, H. M. Kone, J. B. Nikiema, O. G. Nacoulma, and I. P. Guissou, "Evaluation of the antihepatotoxic effect of Argemone mexicana leaf extracts against $\mathrm{CCl}_{4}$-induced hepatic injury in rats," International Journal of Biological and Chemical Sciences, vol. 3, no. 6, 2009.

[50] G. E. B. El Ghazali, W. E. Abdalla, H. E. Khalid, M. M. Khalafalla, and A. A. Hamad, Medicinal Plants of the Sudan Part V. Medicinal Plants of Ingassana Area, National Centre for Research., Khartoum, Sudan, 2003.

[51] T. Okpekon, S. Yolou, C. Gleye et al., "Antiparasitic activities of medicinal plants used in Ivory Coast," Journal of Ethnopharmacology, vol. 90, no. 1, pp. 91-97, 2004.

[52] D. Chothani and H. Vaghasiya, "A review on Balanites aegyptiaca Del (desert date): phytochemical constituents, traditional uses, and pharmacological activity," Pharmacognosy Reviews, vol. 5, no. 9, p. 55, 2011.

[53] S. Ali, A. H. Mohamed, G. E. Mohammed, and A. A. Gameel, "Toxicity of Balanites aegyptiaca seeds oil in wistar albino rats," SUST Journal of Agricultural and Veterinary Sciences, vol. 18, no. 1, 2017.

[54] F. W. Andrews, Andrews, The Flowering Plants of the AnglogyptianSudan, vol. 2, T. Buncle \& Co. Ltd., Arbroath, Scotland, 1952.

[55] A. Bilal, N. Jahan, A. Ahmed, S. Naaz Bilal, S. Habib, and S. Hajra, "Phytochemical and pharmacological studies on Ocimum basilicum linn-a review," International Journal of Current Research and Review, vol. 4, no. 23, 2012.

[56] Y. J. Hong, F. A. Tomas-Barberan, A. A. Kader, and A. E. Mitchell, "The flavonoid glycosides and procyanidin composition of deglet noor dates (phoenix dactylifera)," Journal of Agricultural and Food Chemistry, vol. 54, no. 6, pp. 2405-2411, 2006.

[57] F. W. Andrews, The Flowering Plants of the Anglo EgyptianSudan, vol. 3, T. Buncle \& Co. Ltd., Arbroath, Scotland, 1956.

[58] M. Z. Zaruwa, N. I. Ibok, I. U. Ibok et al., "Effects of Sterculia setigera Del. Stem bark extract on hematological and biochemical parameters of wistar rats," Biochemistry Insights, vol. 9, Article ID BCI.S36143, 2016.

[59] K. Roosita, C. M. Kusharto, M. Sekiyama, Y. Fachrurozi, and R. Ohtsuka, "Medicinal plants used by the villagers of a Sundanese community in West Java, Indonesia," Journal of Ethnopharmacology, vol. 115, no. 1, pp. 72-81, 2008.

[60] M. J. Patil, B. P. Pimple, P. V. Kadam, N. S. Badgujar, and A. R. Bafna, "Protective effect of Tamarindus indica linn against paracetamol-induced hepatotoxicity in rats," Indian Journal of Pharmaceutical Sciences, vol. 69, no. 6, p. 827, 2007.

[61] G. Ai, Q. Liu, W. Hua, Z. Huang, and D. Wang, "Hepatoprotective evaluation of the total flavonoids extracted from flowers of Abelmoschus manihot (L.) Medic: in vitro and in vivo studies," Journal of Ethnopharmacology, vol. 146, no. 3, pp. 794-802, 2013.

[62] S. S. Saboo, G. Tapadiya, I. A. Farooqui, and S.S. Khadabadi, "Free radical scavenging, in vivo antioxidant and hepatoprotective activity of folk medicine Trichodesma sedgwickianum," Bangladesh Journal of Pharmacology, vol. 8, no. 1, pp. 58-64, 2013.

[63] Q. L. Tran, I. K. Adnyana, Y. Tezuka et al., "Hepatoprotective effect of majonoside R2, the major saponin from Vietnamese ginseng (Panax vietnamensis)," Planta Medica, vol. 68, no. 5, pp. 402-406, 2002.

[64] Y. Wang, Z. Lou, Q.-B. Wu, and M.-L. Guo, "A novel hepatoprotective saponin from Celosia cristata L.," Fitoterapia, vol. 81, no. 8, pp. 1246-1252, 2010.

[65] A. Ramadan, F. Harraz, and S. El-Mougy, "Anti-inflammatory, analgesic and antipyretic effects of the fruit pulp of Adansonia digitata," Fitoterapia, vol. 65, p. 418, 1994. 
[66] A. A. Al-Qarawi, M. A. Al-Damegh, and S. A. El-Mougy, "Hepatoprotective influence of Adansonia digitata Pulp," Journal of Herbs, Spices \& Medicinal Plants, vol. 10, no. 3, pp. 1-6, 2003.

[67] P. Latha, S. R. Suja, A. Abraham, S. Rajasekharan, and K. R. Pannikar, "Hepatoprotective effects of Ixora coccinea flower extract on rats," Journal of Tropical Medicinal Plants, vol. 4, no. 1, pp. 33-38, 2003.

[68] B. Victor and A. Grace, "Phytochemical studies, in-vitro antibacterial activities and antioxidant properties of the methanolic and ethyl acetate extracts of the leaves of Anogeissus leiocarpus," International Journal of Biochemistry Research \& Review, vol. 3, no. 2, pp. 137-145, 2013.

[69] A. H. Arbab, "Review on Anogeissus leiocarpus a potent African traditional drug," International Journal of Research in Pharmaceutical Sciences, vol. 4, no. 3, pp. 496-500, 2014.

[70] O. O. Ojo, O. Ladeji, L. Olayaki, and C. C. Jide-Ojo, "Hepatoprotective and antioxidant efficacy of aqueous stem bark extracts of Balanites aegyptiaca (Linn.) Del. against acetaminophen induced liver injury in rats," Journal of Medicinal Herbs and Ethnomedicine, vol. 1, no. 1, pp. 89-96, 2015.

[71] T. M. G. Suky, B. Parthipan, C. Kingston, V. R. Mohan, and P. Tresina Soris, "Hepatoprotective and antioxidant effect of Balanites aegyptiaca (L.) Del against $\mathrm{CCL}_{4}$ induced hepatotoxicity in rats," International Journal of Pharmaceutical Sciences and Research, vol. 2, no. 4, p. 887, 2011.

[72] M. Al-Yahya, "Phytochemical studies of the plants used in traditional medicine of Saudi Arabia," Fitoterapia, vol. 57, no. 3, pp. 179-182, 1986.

[73] S. Pattanayak and S. Priyashree, "Hepatoprotective activity of the leaf extracts from Dendrophthoe falcata (Lf) Ettingsh against carbon tetrachloride-induced toxicity in wistar albino rats," Pharmacognosy Magazine, vol. 4, no. 15, pp. 218-222, 2008.

[74] T. Satyanarayana, A. Anjana, and P. Vijetha, "PHCOG REV.: plant review phytochemical and pharmacological review of some Indian Capparis species," Pharmacognosy Reviews [Phcog Rev.]-Supplement, vol. 2, no. 4, pp. 36-45, 2008.

[75] A. Duhan, B. M. Chauhan, and D. Punia, "Nutritional value of some non-conventional plant foods of India," Plant Foods for Human Nutrition, vol. 42, no. 3, pp. 193-200, 1992.

[76] L. E. A. Hassan, M. B. Ahamed, A. S. Majid et al., "Correlation of antiangiogenic, antioxidant and cytotoxic activities of some Sudanese medicinal plants with phenolic and flavonoid contents," BMC Complementary and Alternative Medicine, vol. 14, no. 1, p. 406, 2014.

[77] S. Sanni, I. Thilza, M. Ahmed, MuhammedTalle, and G. O. Okwor, "The effect of aqueous leaves extract of henna (Lawsoniainermis) in carbon tetrachloride induced hepatotoxicity in swiss albino mice," Academia Arena, vol. 2, no. 6, pp. 87-89, 2010.

[78] B. Manjunatha, K. L. Mankani, S. M. Vidya, V. Krishna, and Y. N. Manohara, "Hepatoprotective activity of Butea superba against carbon tetrachloride induced hepatic damage in rodents," Pharmacognosy Magazine, vol. 4, pp. S41-S45, 2008.

[79] A. Diallo, K. Eklu-Gadeg, T. Mobio et al., "Protective effect of Moringa oleifera Lam. and Lannea kerstingii extracts against cadmium and ethanol-induced lipid peroxidation," Journal of Pharmacology and Toxicology, vol. 4, no. 4, pp. 160-166, 2009.

[80] F. Anwar, S. Latif, M. Ashraf, and A. H. Gilani, "Moringa oleifera: a food plant with multiple medicinal uses," Phytotherapy Research, vol. 21, no. 1, pp. 17-25, 2007.

[81] R. Meera, P. Devi, B. Kameswari, B. Madhumitha, and N. J. Merlin, "Antioxidant and hepatoprotective Activities of
Ocimum basilicum Linn. and Trigonella foenum-graecum Linn. against $\mathrm{H}_{2} \mathrm{O}_{2}$ and $\mathrm{CCl}_{4}$ induced hepatotoxicity in goat liver," Indian Journal of Experimental Biology, vol. 47, no. 7, pp. 584-590, 2009.

[82] A. Mushtaq and M. Ahmad, "Hepatoprotective activity of aqueous-ethanolic extract of Solanum nigrum against nimesulide intoxicated albino rats," European Journal of Zoological Research, vol. 2, no. 2, pp. 19-25, 2013.

[83] M. Marzouk, A. A. Sayed, and A. M. Soliman, "Hepatoprotective and antioxidant effects of Cichorium endivia L. leaves extract against acetaminophen toxicity on rats," Journal of Medicine and Medical Sciences, vol. 2, no. 12, pp. 1273-1279, 2011.

[84] S. A. El-Mougy, S. A. Abed Aziz, M. Al-Shanawany, and A. Omar, "The gonadotropic activity of Palmae in mature male rats," Alexandria Journal of Pharmaceutical Sciences, vol. 5, pp. 156-159, 1991.

[85] M. Kowalska, M. Brandt, and D. Puett, "Inhibition of cytochrome P-450 aromatase activity by plant extracts," Planta Medica, vol. 56, no. 6, p. 675, 1990.

[86] S. Daniyan and H. Muhammad, "Evaluation of the antimicrobial activities and phytochemical properties of extracts of Tamaridus indica against some diseases causing bacteria," African Journal of Biotechnology, vol. 7, no. 14, 2008.

[87] M. S. Musa, F. E. Abdelrasool, E. A. Elsheikh, L. A. M. N. Ahmed, A. L. E. Mahmoud, and S. M. Yagi, "Ethnobotanical study of medicinal plants in the blue nile state, south-eastern Sudan," Journal of Medicinal Plants Research, vol. 5, no. 17, pp. 4287-4297, 2011.

[88] H. H. El-Kamali, "Ethnopharmacology of medicinal plants used in North kordofan (western Sudan)," Ethnobotanical Leaflets, vol. 13, pp. 89-97, 2009. 


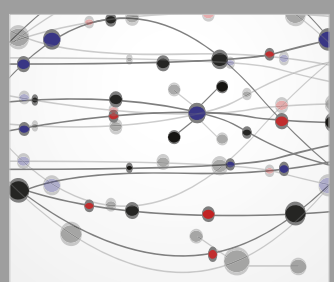

The Scientific World Journal
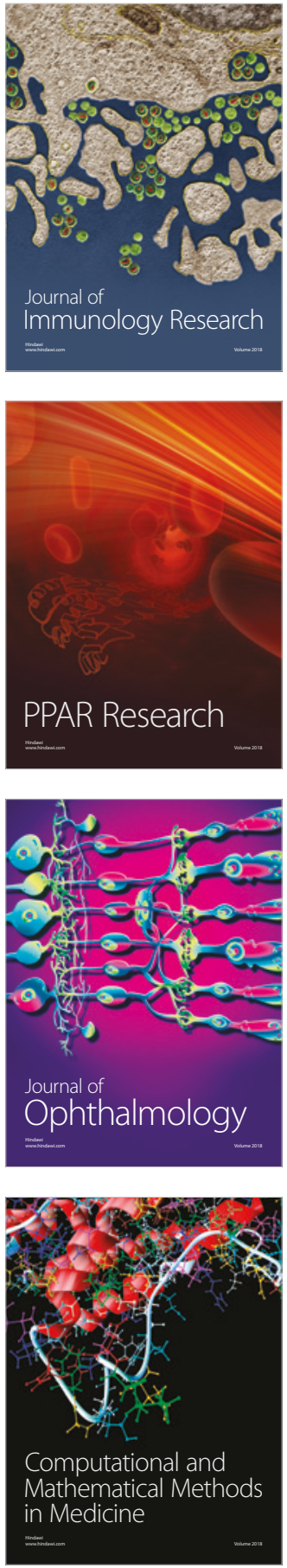

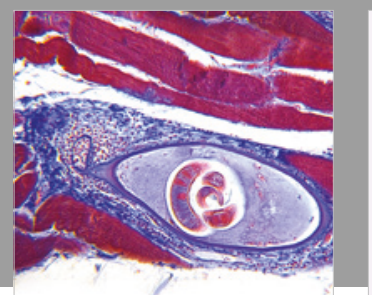

Gastroenterology Research and Practice

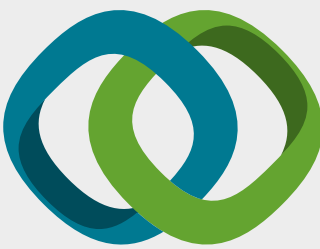

\section{Hindawi}

Submit your manuscripts at

www.hindawi.com
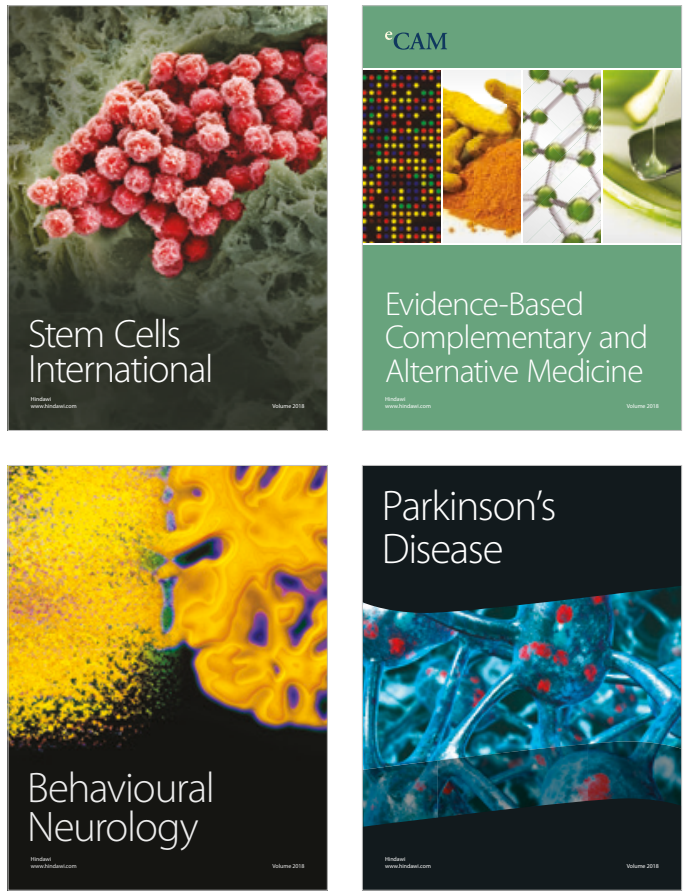

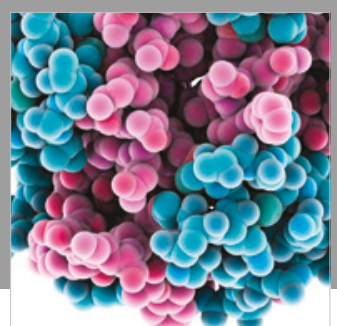

ournal of

Diabetes Research

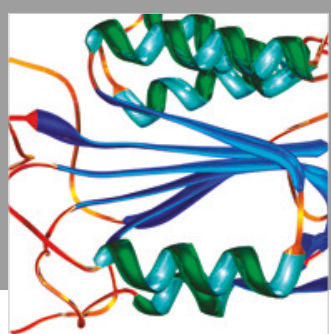

Disease Markers
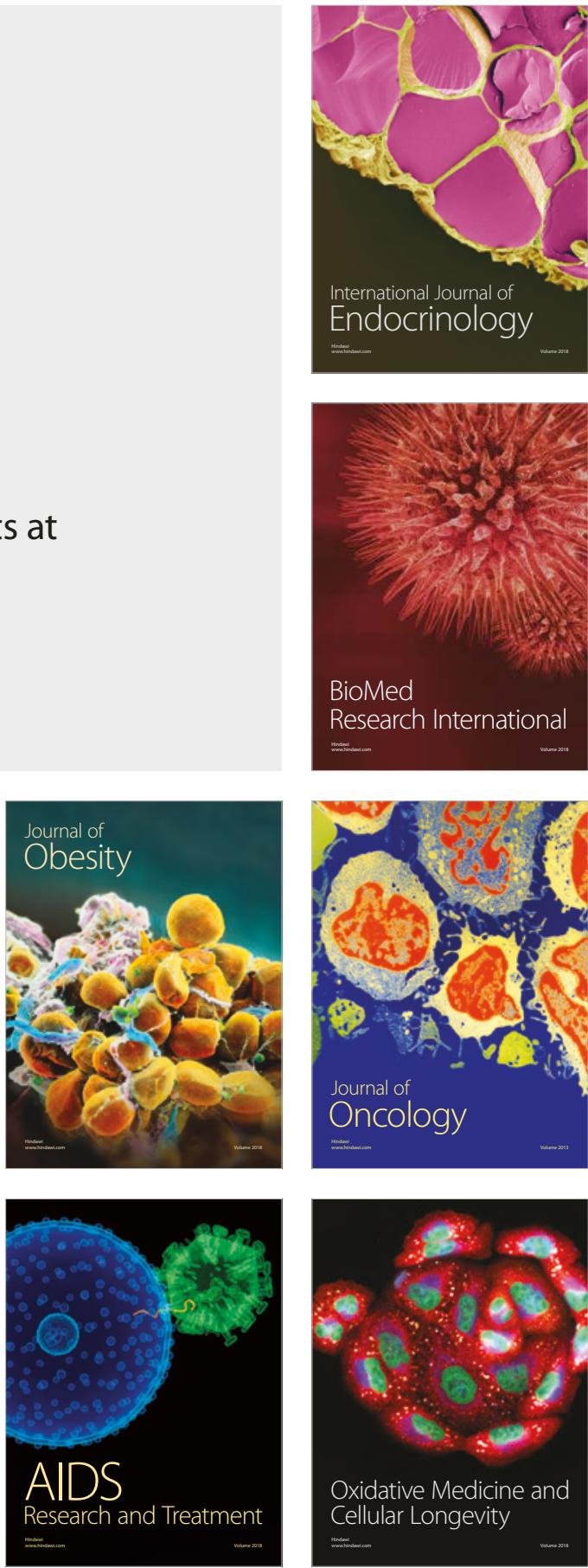